\title{
Tight Convex Relaxations for Vector-Valued Labeling*
}

\author{
Bastian Goldluecke ${ }^{\dagger}$, Evgeny Strekalovskiy $\ddagger$, and Daniel Cremers ${ }^{\ddagger}$
}

\begin{abstract}
Multilabel problems are of fundamental importance in computer vision and image analysis. Yet, finding global minima of the associated energies is typically a hard computational challenge. Recently, progress has been made by reverting to spatially continuous formulations of respective problems and solving the arising convex relaxation globally. In practice this leads to solutions which are either optimal or within an a posteriori bound of the optimum. Unfortunately, in previous methods, both run time and memory requirements scale linearly in the total number of labels, making these methods very inefficient and often not applicable to problems with higher dimensional label spaces. In this paper, we propose a reduction technique for the case that the label space is a continuous product space and the regularizer is separable, i.e., a sum of regularizers for each dimension of the label space. In typical real-world labeling problems, the resulting convex relaxation requires orders of magnitude less memory and computation time than previous methods. This enables us to apply it to large-scale problems like optic flow, stereo with occlusion detection, segmentation into a very large number of regions, and joint denoising and local noise estimation. Experiments show that despite the drastic gain in performance, we do not arrive at less accurate solutions than the original relaxation. Using the novel method, we can for the first time efficiently compute solutions to the optic flow functional which are within provable bounds (typically $5 \%$ ) of the global optimum.
\end{abstract}

Key words. multilabel problems, algorithms, duality, convex relaxation

AMS subject classifications. 68U10, 49M29, 65K10

DOI. $10.1137 / 120862351$

\section{Introduction.}

1.1. The multilabeling problem. Recently, there has been a surge of research activity on convex relaxation techniques for energy minimization in computer vision. Particular efforts have been directed toward binary and multilabel problems, as they lie at the heart of fundamental problems like segmentation [11, 22, 9, 41], stereo [27], three-dimensional reconstruction [12], Mumford-Shah denoising [26], and optic flow [15].

The aim is to assign to each point $x$ of a domain $\Omega \subset \mathbb{R}^{n}$ a label from a set $\Gamma \subset \mathbb{R}^{d}$. Assigning the label $\gamma \in \Gamma$ to $x$ is associated with the $\operatorname{cost}^{\gamma}(x)=c(x, \gamma) \in \mathbb{R}$. In computer vision applications, this local cost denotes how well a given labeling fits some observed data. The cost functions can be arbitrarily sophisticated, derived from statistical models or complicated local matching scores. In the following, we will assume that the cost functions $c^{\gamma}$ lie in

\footnotetext{
${ }^{*}$ Received by the editors January 17, 2012; accepted for publication (in revised form) March 27, 2013; published electronically August 22, 2013. Earlier versions of this paper appeared in the conference proceedings [15] and [36]. This work was supported by the ERC Starting Grant "Convex Vision."

http://www.siam.org/journals/siims/6-3/86235.html

${ }^{\dagger}$ Heidelberg Collaboratory for Image Processing, Heidelberg 69115, Germany (bastian.goldluecke@iwr.uniheidelberg.de).

${ }^{\ddagger}$ Department of Computer Science, Technical University Munich, Garching D-85748, Munich, Germany (evgeny. strekalovskiy@in.tum.de, cremers@in.tum.de).
} 

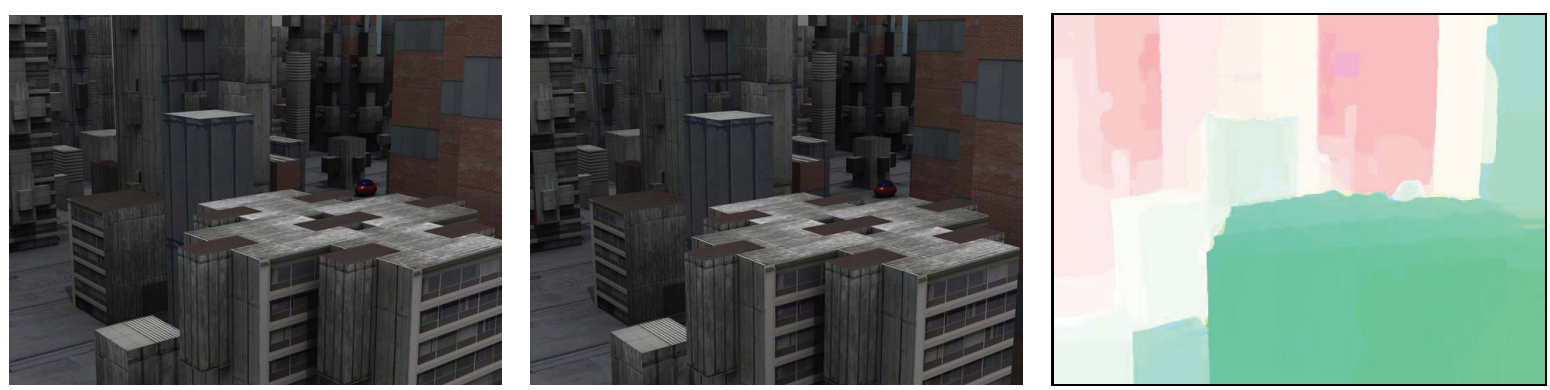

Figure 1. The proposed relaxation method can approximate the solution to multilabeling problems with a huge number of possible labels by globally solving a convex relaxation model. This example shows two images and the optic flow field between them, where flow vectors were assigned from a possible set of $50 \times 50$ vectors, with truncated linear distance as a regularizer. The problem has so many different labels that a solution cannot be computed by alternative relaxation methods on current hardware. Reprinted with permission from [15, Figure 1], (c) 2010 by Springer-Verlag, Berlin, Heidelberg.

the Hilbert space of square integrable functions $\mathcal{L}^{2}(\Omega)$. Aside from minimizing the local costs, we want the optimal assignment to exhibit a certain regularity. We enforce this requirement by penalizing each possible labeling $\boldsymbol{u}: \Omega \rightarrow \Gamma$ with a regularizer $J(\boldsymbol{u}) \in \mathbb{R}$. This regularizer reflects our knowledge about which label configurations are a priori more likely and typically enforces a form of spatial coherence of the computed labeling.

Finding a labeling $\boldsymbol{u}: \Omega \rightarrow \Gamma$ which minimizes the sum of data term and regularizer, i.e.,

$$
\underset{\boldsymbol{u} \in \mathcal{L}^{2}(\Omega, \Gamma)}{\operatorname{argmin}}\left\{J(\boldsymbol{u})+\int_{\Omega} c(x, \boldsymbol{u}(x)) \mathrm{d} x\right\},
$$

is a hard computational challenge as the overall energy is generally not convex. For some cases, good results may be obtained by local minimization, starting from a good initialization, possibly further improved by coarse-to-fine strategies commonly employed in optical flow estimation. Yet, such methods cannot guarantee any form of quality of the result, and performance typically depends on data, on initialization, and on the choice of algorithmic minimization scheme (number of levels in the coarse-to-fine hierarchy, number of iterations per level, etc.). The goal of this paper is to develop solutions to such problems which do not depend on initialization and which lie within a computable bound of the global optimum; see Figure 1.

1.2. Contribution: Product label spaces. In this work, we consider label spaces which can be written as a product of a finite number $d$ of spaces, $\Gamma=\Lambda_{1} \times \cdots \times \Lambda_{d}$. The central idea is as follows. Assume that the spaces $\Lambda_{k}$ are discrete or have been discretized, and let $N_{k}$ be the number of elements in $\Lambda_{k}$. Then the total number of labels is $N=N_{1} \times \cdots \times N_{d}$. In previous relaxations for the multilabel problem, this means that we need to optimize over a number of $N$ binary indicator functions, which can easily amount to thousands of indicator functions in practical problems. In order to make problems of this form feasible to solve, we present a reduction method which requires only $N_{1}+\cdots+N_{d}$ binary functions. As a consequence, memory grows linearly (rather than exponentially) in the number of dimensions, while computation time is greatly reduced. 
An important limitation, however, is that we consider only separable regularizers of the form

$$
J(\boldsymbol{u})=\sum_{k=1}^{d} J_{k}\left(u_{k}\right),
$$

which means that $J$ acts on the label components $u_{k}$ of $\boldsymbol{u}$ independently.

We will show that with the novel reduction technique, it is possible to efficiently solve convex relaxations to multilabel problems which are far too large to approach with existing techniques. A prototypical example is optic flow, where the total number of labels is typically around $32^{2}$ for practical problems. In that case, for example, we require only 64 indicator functions instead of 1024. However, the proposed method applies to a much larger class of labeling problems. This reduction in variable size not only allows for substantially higher resolution of the label space, but also gives rise to a drastic speedup.

The present paper subsumes and extends two previous conference publications $[15,36]$. For this journal paper, both earlier works were integrated into a comprehensive exposition of continuous vectorial labeling problems. Contents of the earlier paper [15] were revised in light of the new findings and improved relaxations in [36], and the differences between both approaches were illuminated. Compared to the original conference publications, the present paper provides a more detailed background and more complete theory. We included the total cyclic variation [35] as an additional regularizer and extended the description of the algorithm we use to implement the method. In particular, we were able to prove that our new data term relaxation corresponds to the exact convex envelope for the nonconvex data term of the energy, which makes it the tightest possible relaxation for the data term. All experiments in the earlier paper were redone and updated with respect to the novel implementation of the algorithm in [36]. We also included a few more more experimental comparisons, in particular, to discrete methods, and extended the implementation to arbitrary label space dimension. Complete source code to reproduce the experiments is publicly available on Sourceforge under a GPL3 license as part of our CUDA library for continuous convex optimization in image processing. ${ }^{1}$

\section{Related work.}

2.1. Discrete approaches. It is well known that in the fully discrete setting, the minimization problem (1.1) is equivalent to maximizing a Bayesian posterior probability, where the prior probability gives rise to the regularizer [37]. The problem can be stated in the framework of Markov random fields (MRFs) and discretized using a graph representation, where the nodes denote discrete pixel locations and the edge weights encode the energy functional [4].

Fast combinatorial minimization methods based on graph cuts can then be employed to search for a minimizer. In the case that the label space is binary and the regularizer submodular, a global solution of (1.1) can be found by computing a minimum cut [16, 21]. Continuous variants of this minimum cut problem have also been studied [34]. For multilabel problems, one can approximate a solution for example by solving a sequence of binary problems ( $\alpha$-expansions) [6,31], linear programming (LP) relaxations [40], or quadratic pseudo-Boolean optimization [20]. Exact solutions to multilabel problems can only be found in some special

\footnotetext{
${ }^{1}$ See https://sourceforge.net/p/cocolib.
} 
cases. An important case are multilabel problems over a linearly ordered label set with convex regularizer. The global optimum of this problem corresponds to a cut in a multilayered graph which can be computed in polynomial time [17]. A different discrete encoding scheme for this problem was also presented in [31].

In $[32,33]$ the problem of image registration is formulated as an MRF labeling problem, which is minimized via LP relaxation. The authors present a decoupling strategy for the displacement components which is related to ours, albeit applicable only in the discrete case. It allows a simplification of the graph and consequently larger numbers of labels. Another discrete method which is related to our work is found in [29]. The authors present a compact encoding scheme for the multilabel problem called a log-transformation which makes the unary term nonsubmodular. This is analogous to our transformation, which makes the previously convex data term nonconvex. The problem of large label spaces is also tackled in [14], where the authors compute optical flow from an MRF labeling problem using a lower dimensional parametric description for the displacements.

However, in many important scenarios the label space cannot be ordered. Moreover, a nonconvex regularizer is often more desirable to better preserve discontinuities in the solution. Even for relatively simple nonconvex regularizers like the Potts distance, the resulting combinatorial problem is NP-hard [6]. In this paper, we work in a spatially continuous setting, avoiding typical problems of graph-based discretization like anisotropy and metrication errors [18].

2.2. Continuous approaches. Continuous approaches address the multilabel problem by means of convex relaxation. To this end, the original nonconvex energy is replaced with a convex lower bound, which can be minimized globally. We automatically get a bound on the solution and know how far we are at most from the global optimum. How good the bound is depends on the tightness of the relaxation, i.e., how close the energy is to the convex envelope relaxation. For particular labeling problems, this strategy even leads to globally optimal solutions. For example, as in the discrete setting, it is possible to solve the two-label problem with length regularity, i.e., the regularizer being equal to the length of the interface between the two regions, in a globally optimal way [11].

The framework presented in this paper is based on the calibration or lifting idea for the Mumford-Shah functional, which was analyzed in depth in $[1,2]$. The idea is that rather than optimizing for the original labeling function, one instead estimates the characteristic function of its epigraph (called the subgraph in [2]). Thus, one ends up with a relaxation of the original problem in terms of these characteristic functions, which is convex. The question is whether the solution of the relaxation corresponds to a solution of the original problem. In $[28,27]$, it was shown that one can achieve a globally optimal solution for the special case of a linearly ordered set of labels and convex regularizers. This construction is related to the discrete approach proposed in [17].

For the general multilabel case, however, there is no relaxation known that would lead to provably optimal solutions. Relaxations of different tightness have been proposed in [22, 9, 41]. They all have in common that they are very memory intensive if the number of labels becomes larger. This makes it impossible to use them for scenarios with thousands of labels, as, for example, optic flow. 
Our previous conference publication [15] relied on a straightforward relaxation of the regularizer proposed in [41] and further generalized in [22], which was designed for a discrete label space. In this paper, we instead employ the relaxation proposed in [25], which is based on the calibration method [2]. Not only is it provably tighter than the above, but it also allows us to accurately represent regularizers for continuous label spaces. In this context, we show that the discrete relaxation $[22,15]$ can also be interpreted as a special case of the more general continuous framework.

\section{Multidimensional label spaces.}

3.1. Discrete product label spaces. From now on we assume that the space of labels is a product of a finite number $d$ of spaces, $\Gamma=\Lambda_{1} \times \cdots \times \Lambda_{d}$. In order to give a more visual explanation of the main idea behind our work, we first discuss the discrete case, where $\left|\Lambda_{k}\right|=N_{k} \in \mathbb{N}$.

The convex relaxation introduced in $[22,41]$ works as follows. Instead of looking for a labeling $\boldsymbol{u}: \Omega \rightarrow \Gamma$ directly, we associate each label $\gamma$ with a binary indicator function $u^{\gamma} \in \mathcal{L}^{2}(\Omega,\{0,1\})$, where $u^{\gamma}(x)=1$ if and only if $\boldsymbol{u}(x)=\gamma$. To make sure that a unique label is assigned to each point, only one of the indicator functions can have the value 1 . We can model this restriction by viewing $\boldsymbol{u}$ as a function mapping into the set of corners $\Delta$ of the $N$-simplex:

$$
\boldsymbol{u} \in \mathcal{L}^{2}(\Omega, \Delta) \text { with } \Delta=\left\{\boldsymbol{x} \in\{0,1\}^{N}: \sum_{j=1}^{N} x_{j}=1\right\} .
$$

Obviously, we can identify $\boldsymbol{u}$ with the vector $\left(u^{\gamma}\right)_{\gamma \in \Gamma}$ of indicator functions. Let $\langle\cdot, \cdot\rangle$ denote the inner product on the Hilbert space $\mathcal{L}^{2}(\Omega)$; then problem (1.1) can thus be written in the equivalent form

$$
\underset{\boldsymbol{u} \in \mathcal{L}^{2}(\Omega, \Delta)}{\operatorname{argmin}}\left\{J(\boldsymbol{u})+\sum_{\gamma \in \Gamma}\left\langle u^{\gamma}, c^{\gamma}\right\rangle\right\},
$$

where we use boldface notation $\boldsymbol{u}$ for vectors $\left(u^{\gamma}\right)_{\gamma \in \Gamma}$ indexed by elements in $\Gamma$. We write $c^{\gamma}(x):=c(x, \gamma)$ for the discrete data term. We also use the same symbol $J$ to denote the regularizer on the reduced space. Its definition requires careful consideration and will be discussed in detail later.

The central idea of the paper is the following. The full discrete label space $\Gamma$ has $N=$ $N_{1} \times \cdots \times N_{d}$ elements, which means that it requires $N$ indicator functions to represent a labeling, one for each label. We will show that it suffices to use $N_{1}+\cdots+N_{d}$ indicator functions, which is a considerable reduction in problem dimensionality, and thus computation time and memory requirements. We achieve this by replacing the indicator functions on the product $\Gamma$ by indicator functions on the components $\Lambda_{k}$.

To this end, we associate to each label $\lambda \in \Lambda_{k}, 1 \leq k \leq d$, an indicator function $v_{k}^{\lambda}$. In each component $k$, only one of the indicator functions can be set. Thus, the vector $\boldsymbol{v}_{k}=\left(v_{k}^{\lambda}\right)_{\lambda \in \Lambda_{k}}$ which consists of $N_{k}$ binary functions can be viewed as a mapping into the corners of the 

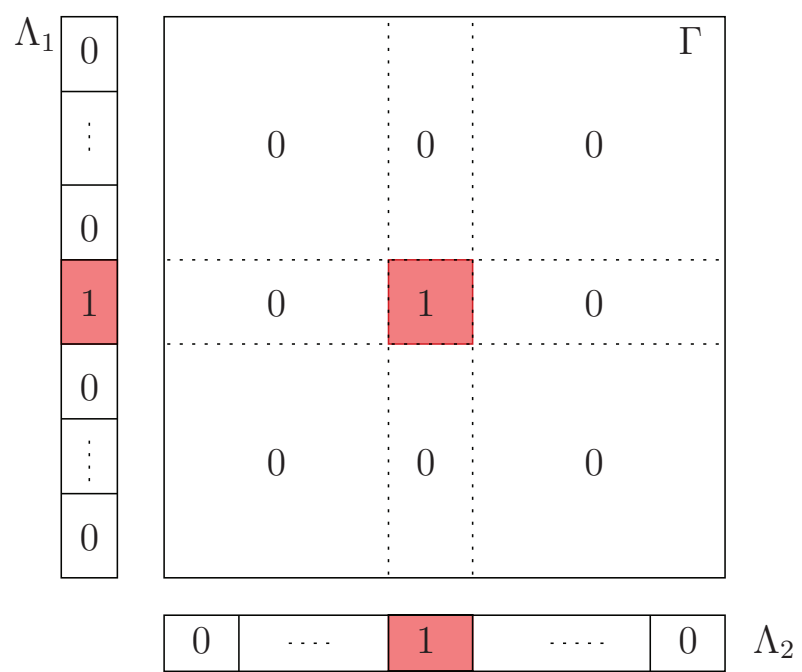

Figure 2. The central idea of the reduction technique is that if a single indicator function in the product space $\Gamma$ takes the value 1 , then this is equivalent to setting an indicator function in each of the factors $\Lambda_{j}$. The memory reduction stems from the fact that there are many more labels in $\Gamma$ than in all the factors $\Lambda_{j}$ combined. Reprinted with permission from [15, Figure 2], (c) 2010 by Springer-Verlag, Berlin, Heidelberg.

$\operatorname{simplex} \Delta_{k}$

$$
\boldsymbol{v}_{k} \in \mathcal{L}^{2}\left(\Omega, \Delta_{k}\right) \text { with } \Delta_{k}=\left\{\boldsymbol{x} \in\{0,1\}^{N_{k}}: \sum_{j=1}^{N_{k}} x_{j}=1\right\} .
$$

In particular, the reduced set of indicator functions $\boldsymbol{v}=\left(v_{k}^{\lambda}\right)_{1 \leq k \leq d, \gamma \in \Lambda_{k}}$ can be seen as a $\operatorname{map} \mathcal{L}^{2}\left(\Omega, \Delta_{\times}\right)$with

$$
\Delta_{\times}=\Delta_{1} \times \cdots \times \Delta_{d} \subset \mathbb{R}^{N_{1}+\cdots+N_{d}} .
$$

Note that an element $\boldsymbol{v} \in \mathcal{L}^{2}\left(\Omega, \Delta_{\times}\right)$consists indeed of exactly $N_{1}+\cdots+N_{d}$ binary functions.

The following proposition illuminates the relationship between the original space of indicator functions $\mathcal{L}^{2}(\Omega, \Delta)$ and the reduced indicator function space $\mathcal{L}^{2}\left(\Omega, \Delta_{\times}\right)$, which is easy to understand visually; see Figure 2.

Proposition 3.1. A bijection $\boldsymbol{v} \mapsto \boldsymbol{u}$ from $\mathcal{L}^{2}\left(\Omega, \Delta_{\times}\right)$onto $\mathcal{L}^{2}(\Omega, \Delta)$ is defined by setting

$$
u^{\gamma}:=v_{1}^{\gamma_{1}} \times \cdots \times v_{d}^{\gamma_{d}} \quad \text { for all } \gamma=\left(\gamma_{1}, \ldots, \gamma_{d}\right) \in \Gamma .
$$

The proof of this proposition, as well as those of the other propositions and theorems in this paper, is given in the appendix.

Using this reduced function space, another formulation equivalent to (1.1) and (3.2) can be given as

$$
\underset{\boldsymbol{v} \in \mathcal{L}^{2}\left(\Omega, \Delta_{\times}\right)}{\operatorname{argmin}}\left\{J(\boldsymbol{v})+\sum_{\gamma \in \Gamma}\left\langle v_{1}^{\gamma_{1}} \times \cdots \times v_{d}^{\gamma_{d}}, c^{\gamma}\right\rangle\right\} .
$$


While we have reduced the dimensionality of the problem considerably, we have introduced another difficulty: the data term is not convex anymore since it contains a product of the components. Thus, in the relaxation, we need to take additional care to make the final problem convex again.

3.2. Continuous label spaces and relaxation framework. We now turn to the more general case that each factor $\Lambda_{k}$ is an interval in $\mathbb{R}$, which means that we deal with a continuous label space with an infinite number of labels. In this situation, one is also interested in a number of continuous regularizers, which cannot be modeled satisfyingly on a discrete label space. As in the discrete case, the regularizers are usually not convex and require a relaxation.

In the context of continuous labeling problems where the label range is an interval, a central idea is functional lifting, which is a variant of the calibration method [2]. Here, one works with characteristic functions describing the hypograph instead of the labeling function itself, an idea that was further refined and applied to a variety of image processing problems in a number of subsequent works [9, 25, 26, 27]. We are going to translate this framework to the case of a product label space. With the regularizer, we can restrict ourselves to the case where it can be decomposed into the sum of regularizers on each component. However, for the data term this is not possible since the cost function usually cannot be decomposed in a similar way. Therefore, we need to define a relaxation framework in which we still can express arbitrary cost functions.

Let us first consider a single component $u_{k}: \Omega \rightarrow \Lambda_{k}$ of the full labeling function $\boldsymbol{u}$. The characteristic function of its hypograph is defined on $\Omega \times \Lambda_{k}$ as

$$
1_{\text {hyp }\left(u_{k}\right)}(x, \lambda)= \begin{cases}1 & \text { if } \lambda<u_{k}(x), \\ 0 & \text { else. }\end{cases}
$$

In $[2,26,27]$, the labeling problem is reformulated in terms of new unknowns which correspond to these characteristic functions. The reason is (4.1), which we discuss later and which allows to give a convex reformulation of the regularizer in terms of the new unknowns. This allows us to obtain a globally optimal solution in the new variables, which often is at least close to and sometimes equal to the solution of the original nonconvex problem.

In our case, however, we need different variables in order to be able to simultaneously formulate a convex relaxation of the data term. We work with the indicator functions denoting whether a specific label $\lambda$ is set at a point $x \in \Omega$, related to a labeling $\boldsymbol{u}$ by

$$
v_{k}(x, \lambda)=\delta\left(u_{k}(x)-\lambda\right),
$$

where $\delta$ is the Dirac distribution. Note that the new unknowns are actually distributions on the higher dimensional space $\Omega \times \Lambda_{k}$, which, however, will reduce to regular functions after discretization. They serve as a generalization of the discrete label indicator functions $\boldsymbol{v}_{k} \in$ $\mathcal{L}^{2}\left(\Omega, \Delta_{k}\right)$ in (3.3) to the continuous case; in particular they satisfy the relations

$$
\int_{\Lambda_{k}} v_{k}(x, \lambda) \mathrm{d} \lambda=1, \quad \int_{\Lambda_{k}} \lambda v_{k}(x, \lambda) \mathrm{d} \lambda=u_{k}(x),
$$

which mimic the discrete case with sums replaced by integrals. Intuitively, this means that for each fixed $x \in \Omega, v_{k}(x, \cdot)$ has a total mass of 1 and is concentrated on the label $u_{k}(x) \in \Lambda_{k}$. 


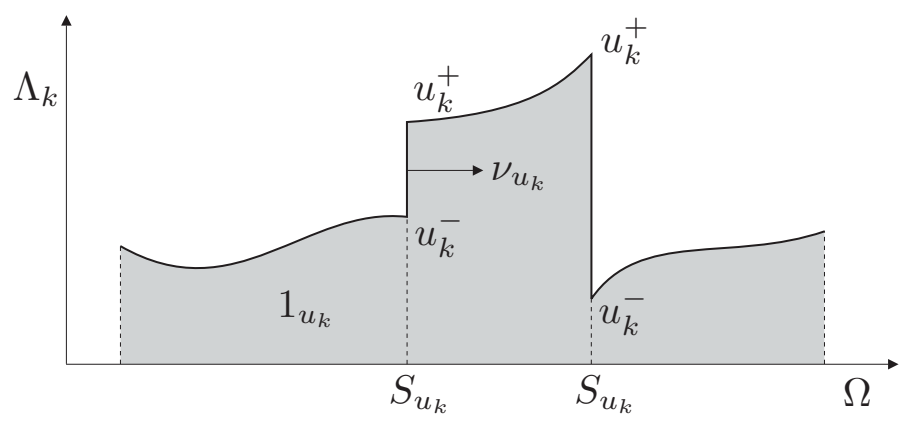

Figure 3. A special function of bounded variation $u$ has an approximate gradient everywhere except on a nullset $S_{u}$, where the values jump from $u^{-}$to $u^{+}$. The normal $\nu_{u}$ denotes the direction of the jump from small to large values. Adapted from Figure 2 in [36].

We will reformulate the labeling problem in terms of the new variables $\boldsymbol{v}$ in section 4 . Some things have to be kept in mind, however. Since the new variables are distributions in the continuous case, we cannot formulate a well-defined minimization problem without first reducing them to $\mathcal{L}^{2}$-functions. This means that before writing the actual minimization problem that we want to solve in the new variables, we have to introduce a discretization of the label space. Despite the necessary discretization, we follow other previous works which employ the lifting idea $[25,26,27,28]$ and still insist that we correctly deal with a continuous label space. This is justified since the definition of the continuous regularizers in section 5 does not make use of the discretization, in contrast to, e.g., [22], where the label space is discretized from the beginning. One thing which remains to be discussed, however, is whether the discrete solutions converge to the continuous one when the label space discretization is refined, in the spirit of [8]. This is a possible avenue for future work.

3.3. Regularization. As stated in the beginning, we consider a separable regularizer of the form

$$
J(\boldsymbol{u})=\sum_{k=1}^{d} J_{k}\left(u_{k}\right) .
$$

In order to define its components, we require some technical preliminaries. Recall [3, Definition 10.5.1] that for functions $u_{k}$ in the space $\mathcal{S B V}(\Omega)$ of special functions of bounded variation, the distributional derivative $D u_{k}$ can be decomposed as

$$
D u_{k}=\nabla u_{k} \mathrm{~d} x+\left(u_{k}^{+}-u_{k}^{-}\right) \nu_{u_{k}} \mathrm{~d} \mathcal{H}^{n-1}\left\llcorner S_{u_{k}}\right.
$$

into a differentiable part and a jump part; see Figure 3. Here, $S_{u_{k}}$ is the $(n-1)$-dimensional jump set of $u_{k}$, where the values jump from $u_{k}^{-}$to $u_{k}^{+}, \nu_{u_{k}}$ is the normal to $S_{u_{k}}$ oriented toward the $u_{k}^{+}$side, and $\nabla u_{k}$ is the approximate gradient of $u_{k}$ [3, Proposition 10.4.1]. The measure $\mathcal{H}^{n-1}\left\llcorner S_{u_{k}}\right.$ is the $(n-1)$-dimensional Hausdorff measure restricted to the set $S_{u_{k}}$. We refer to [3] for a comprehensive introduction to functions of bounded variation.

Making use of this decomposition, we can introduce the framework for regularization. We consider regularizers for problem (1.1) of the form (3.10), with 


$$
J_{k}\left(u_{k}\right)=\int_{\Omega \backslash S_{u_{k}}} h_{k}\left(x, u_{k}(x), \nabla u_{k}(x)\right) \mathrm{d} x+\int_{S_{u_{k}}} d_{k}\left(s, u_{k}^{-}(s), u_{k}^{+}(s)\right) \mathrm{d} \mathcal{H}^{n-1}(s),
$$

with functions $h_{k}: \Omega \times \Lambda_{k} \times \mathbb{R}^{n} \rightarrow \mathbb{R}$ and $d_{k}: \Omega \times \Lambda_{k} \times \Lambda_{k} \rightarrow \mathbb{R}$. The functions $h_{k}$ and $d_{k}$ have to satisfy the following conditions:

1. $h_{k}(x, \lambda, \cdot)$ is convex for fixed $x \in \Omega$ and $\lambda \in \Lambda_{k}$, and $h_{k}(x, \cdot, \cdot)$ is lower semicontinuous for fixed $x \in \Omega$.

2. $d_{k}(x, \cdot, \cdot)$ is a metric on $\Lambda_{k}$ for fixed $x \in \Omega$, and $d_{k}$ is continuous.

The interesting task, of course, is to identify suitable choices of $h_{k}$ and $d_{k}$ and to interpret what the choices mean in practice. We will turn to this in section 5. Before we can explore the possible regularizers, however, we need to introduce a convex relaxation of the general regularizer (3.12) in section 4.

3.4. Notation conventions. Because the label space is multidimensional, the notation requires multiple indices and is slightly more complex. Throughout this work, we keep the following conventions to keep it as clear as possible. The index $k=1, \ldots, d$ enumerating the factors of the product space is always written as a subscript. Indices which are Greek letters always enumerate labels, where $\gamma, \chi$ are labels in the full product space $\Gamma$ with components $\gamma_{k}, \chi_{k} \in \Lambda_{k}$. Greek letters $\lambda, \mu$ denote labels in one of the factors $\Lambda_{k}$. If the label space is discrete or has been discretized, the label is written as a superscript to the indicator functions $v_{k}^{\lambda}$. In the case of a continuous label space, the indicator functions $v_{k}$ live on $\Omega \times \Lambda_{k}$; thus the label appears as an argument of the function $v_{k}(x, \lambda)$.

4. Convex relaxation. The minimization problem (3.2) which we want to solve is not convex: neither is the energy a convex function nor is the domain of minimization a convex set. Thus, the task of finding a global minimizer is in general computationally infeasible. We therefore propose a convex relaxation. This means that instead of minimizing the original functional, we minimize a convex one (ideally, the exact convex envelope) over the convex hull of the original domain.

The relaxation is defined in terms of the new variables $v_{k}$ defined in (3.8). After obtaining a solution $\hat{\boldsymbol{v}}$, the question remains of whether the solution corresponds to a function $\hat{\boldsymbol{u}}$ which solves the original problem. In general, this is not the case, but we can compute a projection $\Pi(\hat{\boldsymbol{v}})$ onto the original problem domain and obtain an optimality bound. Indeed, the energy of the optimal solution $\hat{\boldsymbol{u}}$ must lie somewhere between the energies of $\hat{\boldsymbol{v}}$ and $\Pi(\hat{\boldsymbol{v}})$, as $\hat{\boldsymbol{v}}$ minimizes the relaxation and $\Pi(\hat{\boldsymbol{v}})$ lies in the original problem domain in which $\hat{\boldsymbol{u}}$ is a minimizer.

In the following subsection we will introduce first a convex relaxation of the regularizer, which is based on the calibration method-however, our variables are different from those used in previous work, which requires a slight reformulation. Thereafter, we present the new convex relaxation of the data term and show how it is an improvement over that presented in the original conference paper [15].

4.1. Convex relaxation of the regularizer. Our first goal is to give a new representation of the regularizer defined in (3.12). While in general it is not convex in the labeling $\boldsymbol{u}$, we will obtain a representation which is convex in the new variables $v_{k}$ defined in (3.8). We do this by making use of the calibration or lifting technique described in detail in [2]. 
Lemma 3.9 in [2] states that under the previous assumptions on $h_{k}$ and $d_{k}$, the regularizer $J_{k}$ for each component can be represented as

$$
J_{k}\left(u_{k}\right)=\sup _{\phi \in K}\left\{\int_{\Omega \times \Lambda_{k}} \phi^{1} \cdot \nabla_{x} 1_{\operatorname{hyp}\left(u_{k}\right)}+\phi^{2} \partial_{\lambda} 1_{\operatorname{hyp}\left(u_{k}\right)} \mathrm{d}(x, \lambda)\right\}
$$

with the convex set

$$
\begin{gathered}
K=\left\{\phi=\left(\phi^{1}, \phi^{2}\right) \in C_{c}^{1}\left(\Omega \times \Lambda_{k} ; \mathbb{R}^{n} \times \mathbb{R}\right) \text { such that for all } x \in \Omega \text { and } \lambda, \mu \in \Lambda_{k},\right. \\
\left.\phi^{2}(x, \lambda) \geq h_{k}^{*}\left(x, \lambda, \phi^{1}(x, \lambda)\right) \text { and }\left|\int_{\lambda}^{\mu} \phi^{1}(x, s) \mathrm{d} s\right| \leq d_{k}(x, \lambda, \mu)\right\} .
\end{gathered}
$$

Above, $h_{k}^{*}(x, \lambda, \cdot)$ denotes the convex conjugate of $h_{k}(x, \lambda, \cdot)$. In a slight abuse of notation, the index $c$ in the set $C_{c}^{1}\left(\Omega \times \Lambda_{k} ; \mathbb{R}^{n} \times \mathbb{R}\right)$ indicates that $\phi$ must have compact support, but only with respect to the $x \in \Omega$ variable. Note that (4.1) is a convex representation of the regularizer in terms of the characteristic functions $1_{\mathrm{hyp}\left(u_{k}\right)}$ of the hypograph of $u_{k}$; see (3.7). However, what we want is a convex representation in terms of our new unknowns $v_{k}$. We give this reformulation in the following theorem.

Theorem 4.1. Let $J_{k}$ be of the form (3.12), and let the indicator functions $v_{k}$ be defined as in (3.8). Then

$$
J_{k}\left(u_{k}\right)=\sup _{(\boldsymbol{p}, b) \in C_{k}}\left\{\int_{\Omega \times \Lambda_{k}}(-\operatorname{div}(\boldsymbol{p})-b) v_{k} \mathrm{~d}(x, \lambda)\right\},
$$

with the convex set

$$
\begin{gathered}
C_{k}=\left\{(\boldsymbol{p}, b) \in C_{c}^{1}\left(\Omega \times \Lambda_{k} ; \mathbb{R}^{n} \times \mathbb{R}\right) \text { such that for all } x \in \Omega \text { and } \lambda, \mu \in \Lambda_{k},\right. \\
b(x, \lambda) \geq h_{k}^{*}\left(x, \lambda, \partial_{\lambda} \boldsymbol{p}(x, \lambda)\right), \\
\left.|\boldsymbol{p}(x, \lambda)-\boldsymbol{p}(x, \mu)|_{2} \leq d_{k}(x, \lambda, \mu)\right\} .
\end{gathered}
$$

Note that similarly to the discrete version of the indicator functions, the discrete version of the set $C_{k}$ in (4.4) will consist of tuples $\left(\boldsymbol{p}^{\lambda}, b^{\lambda}\right)_{\lambda \in \Lambda_{k}}$ of functions. Taking a closer look at (4.3), we can see that the right-hand side is a convex functional in the new variables $v_{k}$. Thus, we have achieved our goal and can turn toward finding a similar relaxation of the data term.

4.2. Convex relaxation of the data term. In this subsection, we deal with the nonconvexity of the data term in (3.6),

$$
E_{\text {data }}(\boldsymbol{v})=\sum_{\gamma \in \Gamma}\left\langle v_{1}^{\gamma_{1}} \times \cdots \times v_{d}^{\gamma_{d}}, c^{\gamma}\right\rangle .
$$

Specifically, we show two different ways that it can be replaced with a convex function which coincides with the original data term for binary functions. We first describe the convexification 


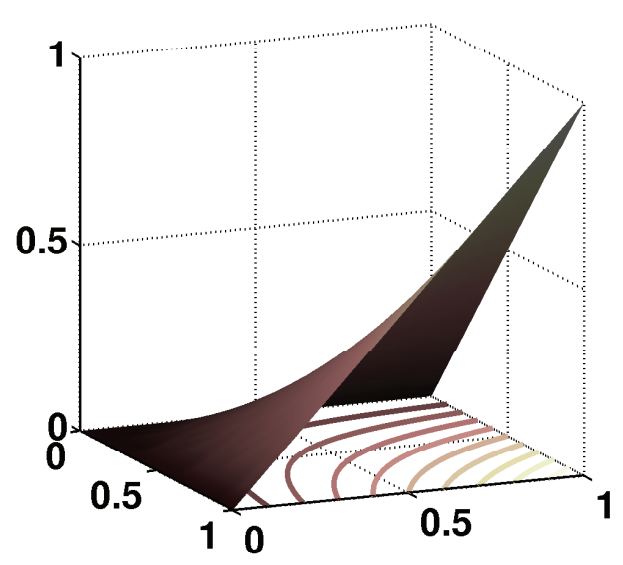

(a) Product function $m\left(x_{1}, x_{2}\right)=x_{1} x_{2}$.

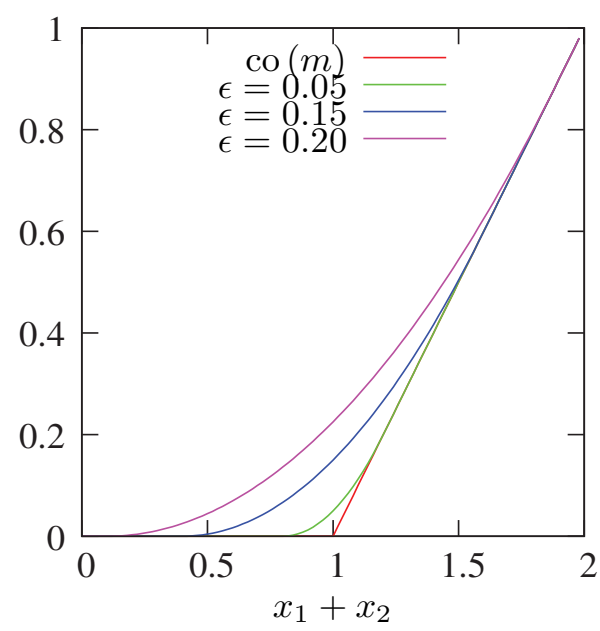

(b) Convex envelope co $(m)$ and mollified versions for different $\epsilon$.

Figure 4. Product function and its mollified convex envelope for the case $d=2$. Reprinted with permission from [15, Figure 3], (c) 2010 by Springer-Verlag, Berlin, Heidelberg.

idea from the original conference paper [15] in the discrete case with a label space of dimension $d=2$. While it leads to a working relaxation, it has certain shortcomings, the main problem being that an unwanted constant solution has to be avoided by additional smoothing when moving on from binary to continuous functions. These shortcomings will be remedied by a new relaxation technique which we explain thereafter. We will show that this relaxation is actually the best possible one, i.e., the convex envelope of the data term. Note that for the data term, we already work in the setting of a discretized label space. While it is possible to give a well-defined theoretical justification of the relaxation for the continuous case, the associated trouble and loss of clarity are not worth the small theoretical gain.

Discrete two-dimensional case. In [15], we suggested replacing the multiplication function $m\left(v_{1}^{\gamma_{1}}, \ldots, v_{d}^{\gamma_{d}}\right):=v_{1}^{\gamma_{1}} \times \cdots \times v_{d}^{\gamma_{d}}$ with its convex envelope co $(m)$. Analyzing the epigraph of $m$ (see Figure 4(a)) shows that

$$
\operatorname{co}(m)\left(v_{1}^{\gamma_{1}}, \ldots, v_{d}^{\gamma_{d}}\right)= \begin{cases}1 & \text { if } v_{1}^{\gamma_{1}}=\cdots=v_{d}^{\gamma_{d}}=1, \\ 0 & \text { if any } v_{k}^{\gamma_{k}}=0 .\end{cases}
$$

This means that if in the functional, $m$ is replaced by the convex function co $(m)$, we retain the same binary solutions, as the function values on binary input are the same.

We lose nothing on first glance, but on second glance, we forfeit differentiability of the data term, since co $(m)$ is not a smooth function any longer. Furthermore, the new function we obtain is not the correct convex envelope of the full data term, but only for the constituting addends. The particular problem this leads to is that for the constant function $\hat{\boldsymbol{v}}$ defined by

$$
\hat{v}_{k}^{\lambda}(x):=1 / N_{k}
$$

the energy of the data term and hence the total energy are zero. 
In [15], this problem was circumvented by an additional mollification of the convex envelope. We replaced $\operatorname{co}(m)$ again by a mollified function $\operatorname{co}(m)_{\epsilon}$, where $\epsilon>0$ is a small constant. We illustrate this for the case $d=2$, where one can easily write down the functions explicitly. In this case, the convex envelope of multiplication is

$$
\operatorname{co}(m)\left(x_{1}, x_{2}\right)= \begin{cases}0 & \text { if } x_{1}+x_{2} \leq 1, \\ x_{1}+x_{2}-1 & \text { otherwise }\end{cases}
$$

This is a piecewise linear function of the sum of the arguments, i.e., symmetric in $x_{1}$ and $x_{2}$; see Figure 4(b). We smoothen the kink by replacing co $(m)$ with smoothed version co $(m)_{\epsilon}$; see [15]. This function does not satisfy the envelope condition (4.6) exactly, but only fulfills the less tight

$$
\operatorname{co}(m)_{\epsilon}\left(x_{1}, \ldots, x_{d}\right) \begin{cases}=1 & \text { if } x_{1}=\cdots=x_{d}=1, \\ \leq \epsilon & \text { if any } x_{j}=0 .\end{cases}
$$

Notably, the data term energy of the constant trivial minimizer (4.7) now becomes $\epsilon \sum_{\gamma} c^{\gamma}$, which means that the relaxation of the data term leads to the correct pointwise solution with energy $\min _{\gamma}\left(c^{\gamma}\right)$ if $\epsilon>\min _{\gamma}\left(c^{\gamma}\right) / \sum_{\gamma} c^{\gamma}$. Since the condition must be satisfied for each point $x \in \Omega$, it is best to let $\epsilon=\epsilon(x)$ depend on $x \in \Omega$ and set it pointwise to the minimal possible value. However, the choice of mollified envelope is suboptimal since it is just an approximation to the correct envelope and distorts the original problem. Thus, we are now going to propose a novel relaxation of the data term which avoids this problem altogether and is easier to deal with in higher dimensional label spaces.

New convex envelope relaxation for the general d-dimensional case. In this paragraph, we describe our new relaxation of the data term. It is the tightest possible relaxation and does not suffer from the described drawbacks of the relaxation in [15]. The new relaxation of $E_{\text {data }}(\boldsymbol{v})$ is one of the main additional contributions of this paper. It is defined as

$$
R_{\text {data }}(\boldsymbol{v}):=\sup _{\boldsymbol{q} \in \mathcal{Q}}\left\{\int_{\Omega} \sum_{\gamma_{1} \in \Lambda_{1}} q_{1}^{\gamma_{1}} v_{1}^{\gamma_{1}}+\cdots+\sum_{\gamma_{d} \in \Lambda_{d}} q_{d}^{\gamma_{d}} v_{d}^{\gamma_{d}} \mathrm{~d} x\right\} .
$$

The additional dual variables $\boldsymbol{q}=\left(\boldsymbol{q}_{k}\right)_{k=1, \ldots, d}$ range over the convex set

$$
\begin{array}{r}
\mathcal{Q}:=\left\{\boldsymbol{q} \in \mathcal{L}^{2}\left(\Omega, \mathbb{R}^{N_{1}+\cdots+N_{d}}\right) \text { such that for all } x \in \Omega \text { and } \gamma \in \Gamma,\right. \\
\left.\qquad q_{1}^{\gamma_{1}}(x)+\cdots+q_{d}^{\gamma_{d}}(x) \leq c^{\gamma}(x)\right\} .
\end{array}
$$

We first establish that the relaxation coincides with the original energy for binary functions.

Proposition 4.2. Let $\boldsymbol{v} \in \mathcal{L}^{2}\left(\Omega, \Delta_{\times}\right)$be a binary function representing the label $\gamma(x) \in \Gamma$ at each point $x \in \Omega$. Then

$$
R_{\text {data }}(\boldsymbol{v})=\int_{\Omega} c^{\gamma(x)}(x) \mathrm{d} x=E_{\text {data }}(\boldsymbol{v})
$$


In addition, one can prove the following theorem, which shows that the relaxation of the data term has the correct pointwise minimizers, in contrast to the one proposed in [15]. This means that no smoothing is necessary and an exact minimization algorithm can be employed to obtain solutions.

Theorem 4.3. Let $\hat{\boldsymbol{v}} \in \mathcal{L}^{2}\left(\Omega, \Delta_{\times}\right)$be a binary minimizer of $E_{\mathrm{data}}$. Then $\hat{\boldsymbol{v}}$ is also a minimizer of the relaxation,

$$
\hat{\boldsymbol{v}} \in \underset{\boldsymbol{v} \in \mathcal{L}^{2}\left(\Omega, \operatorname{co}\left(\Delta_{\times}\right)\right)}{\operatorname{argmin}}\left\{R_{\text {data }}(\boldsymbol{v})\right\}
$$

In particular, $E_{\text {data }}(\hat{\boldsymbol{v}})=R_{\text {data }}(\hat{\boldsymbol{v}})=\int_{\Omega} \hat{c}(x) \mathrm{d} x$ with $\hat{c}(x):=\inf _{\gamma \in \Gamma}\left(c^{\gamma}(x)\right)$ for $x \in \Omega$.

In fact, it turns out that the proposed data term relaxation is the best possible one, being the convex envelope of the data term as stated in the proposition below. More specifically, this is up to the natural sum equality $\sum_{\lambda \in \Lambda_{k}} v_{k}^{\lambda}=1$, which is the first equation in (3.9) and is also used in the definition (6.1) of the $\boldsymbol{v}$ domain in the overall optimization problem. To make this statement precise, we first need a general definition of the data term $E_{\text {data }}$ for all indicator functions $\boldsymbol{v} \in \mathcal{L}^{2}\left(\Omega, \mathbb{R}^{N_{1}+\cdots+N_{d}}\right)$, not only for binary ones. If $\boldsymbol{v}$ is binary representing the label $\gamma(x) \in \Gamma$ at each $x \in \Omega$, i.e., $v_{k}^{\lambda}(x)=\chi_{\lambda=\gamma_{k}(x)}, E_{\text {data }}$ is already defined by (4.5) as

$$
E_{\text {data }}(\boldsymbol{v})=\int_{\Omega} c^{\gamma(x)}(x) \mathrm{d} x=\int_{\Omega} \sum_{\gamma \in \Gamma} c^{\gamma}(x) v_{1}^{\gamma_{1}}(x) \cdots v_{d}^{\gamma_{d}}(x) \mathrm{d} x .
$$

For all other $\boldsymbol{v}$ we set $E_{\text {data }}(\boldsymbol{v}):=\infty$.

Proposition 4.4. The convex envelope of $E_{\mathrm{data}}(\boldsymbol{v})$ is given by $R_{\mathrm{data}}(\boldsymbol{v})+\delta_{\mathcal{S}}(\boldsymbol{v})$ with $\mathcal{S}:=$ $\left\{\boldsymbol{v} \mid \sum_{\lambda \in \Lambda_{k}} v_{k}^{\lambda}(x)=1\right.$ for all $\left.1 \leq k \leq d, x \in \Omega\right\}$.

5. Multilabel regularizers. In this section, we will explore suitable choices of the regularizer and how they fit within the proposed framework. In particular, we will see how our model can be specialized to the case of discrete label spaces where the label distance has a Euclidean representation. This special case was discussed in [22, 15], and we will see that our framework leads to a tighter relaxation for this case. We will also discuss additional continuous regularizers which become possible based on the lifting framework discussed in the last section. These were introduced in the previous works $[9,26,27]$ when the unknowns were the characteristic functions of the hypographs of $u_{k}$. We show how we can accommodate them to depend on the new unknowns. Notably, in each dimension of the label space its own type of regularization can be chosen; in particular discrete and continuous regularizers can be mixed freely.

5.1. Discrete label space and its Euclidean representation. We first consider the special case of a discrete label space $\Lambda_{k}$. Thus, we need to define a regularizer $J_{k}: \mathcal{L}^{2}\left(\Omega, \operatorname{co}\left(\Delta_{k}\right)\right) \rightarrow \mathbb{R}$ for functions with values in the convex hull of the simplex $\Delta_{k}$. We first present the construction used in $[22,15]$ and then show how we can embed it into our more general framework.

We assume that the metric $d_{k}$ has a Euclidean representation. This means that each label $\lambda \in \Delta_{k}$ shall be represented by an $M_{k}$-dimensional vector $\boldsymbol{a}_{k}^{\lambda} \in \mathbb{R}^{M_{k}}$ with an $M_{k} \geq 1$, and the distance $d_{k}$ is defined as the Euclidean distance between the representations, 


$$
d_{k}(\lambda, \mu)=\left|\boldsymbol{a}_{k}^{\lambda}-\boldsymbol{a}_{k}^{\mu}\right|_{2} \quad \text { for all } \lambda, \mu \in \Delta_{k}
$$

The goal in the construction of $J_{k}$ is that the greater the distance between labels and the longer the jump set, the greater shall be the penalty imposed by $J_{k}$. To make this idea precise, we introduce the linear mappings $A_{k}: \operatorname{co}\left(\Delta_{k}\right) \rightarrow \mathbb{R}^{M_{k}}$ which map labels onto their representations,

$$
A_{k}(\lambda)=\boldsymbol{a}_{k}^{\lambda} \text { for all } \lambda \in \Delta_{k}
$$

When the labels are enumerated and represented by the indicator functions $\boldsymbol{v}_{k}$ in (3.3), then in matrix notation, the vectors $\boldsymbol{a}_{k}^{\lambda}$ become exactly the columns of $A_{k}$, which shows the existence of this map. It turns out that a regularizer with desirable properties can be defined by

$$
J_{k}^{A}\left(\boldsymbol{v}_{k}\right):=\mathrm{TV}_{\mathrm{v}}\left(A_{k} \boldsymbol{v}_{k}\right),
$$

where

$$
\operatorname{TV}_{\mathrm{v}}(\boldsymbol{f}):=\int_{\Omega} \sqrt{\sum_{i=1}^{m}\left|\nabla f_{i}\right|_{2}^{2}} \mathrm{~d} x
$$

denotes the vectorial total variation (TV) for functions $f: \Omega \rightarrow \mathbb{R}^{m}$ taking values in a real vector space of dimension $m$. The following theorem has been proved in [22] and shows why the above definition makes sense.

Theorem 5.1. The regularizer $J_{k}^{A}$ defined in (5.3) has the following properties:

1. $J_{k}^{A}$ is convex and positively homogeneous on $\mathcal{L}^{2}\left(\Omega, \operatorname{co}\left(\Delta_{k}\right)\right)$.

2. $J_{k}^{A}\left(\boldsymbol{v}_{k}\right)=0$ for any constant labeling $\boldsymbol{v}_{k}$.

3. If $S \subset \Omega$ has finite perimeter $\operatorname{Per}(S)$, then for all labels $\lambda, \mu \in \Lambda_{k}$,

$$
J_{k}^{A}\left(\lambda 1_{S}+\mu 1_{S^{c}}\right)=d_{k}(\lambda, \mu) \operatorname{Per}(S) ;
$$

i.e., a change in labels is penalized proportionally to the distance between the labels and the perimeter of the interface.

For the sake of simplicity, we give the main examples only for distances with Euclidean representations. More general classes of distances on the labels can also be used; see [22].

- There is the case of ordered labels, where the embedding follows the natural ordering of $\lambda, \mu \in \mathbb{R}$ (Figure $5(\mathrm{a})$ ), for example, by setting simply $\boldsymbol{a}_{k}^{\lambda}=\lambda$. If $d=1$, then this case can be solved in a globally optimal way using the lifting method [27].

- Another case is the Potts or uniform distance, where $d_{k}(\lambda, \mu)=1$ if and only if $\lambda=\mu$, and zero otherwise. This distance function can be achieved by setting $\boldsymbol{a}_{k}^{\lambda}=\frac{1}{\sqrt{2}} e^{\lambda}$, where $\left(\boldsymbol{e}^{\lambda}\right)_{\lambda \in \Lambda_{k}}$ is an orthonormal basis in $\mathbb{R}^{N_{k}}$; see Figure $5(\mathrm{~b})$. All changes between labels are penalized equally.

- Another typical case is that the $\boldsymbol{a}_{k}^{\lambda}$ denote feature vectors or actual geometric points, for which $|\cdot|_{2}$ is a natural distance. For example, in the case of optic flow, each label corresponds to a flow vector in $\mathbb{R}^{2}$; see Figure $5(\mathrm{c})$. The representations $\boldsymbol{a}_{1}^{\lambda}, \boldsymbol{a}_{2}^{\mu}$ are just real numbers, denoting the possible components of the flow vectors in the $x$ - and 


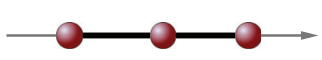

(a) Ordered embedding

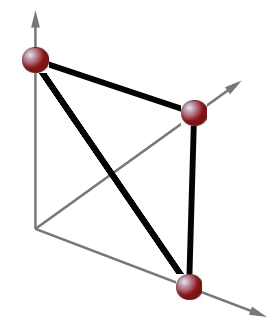

(b) Potts embedding

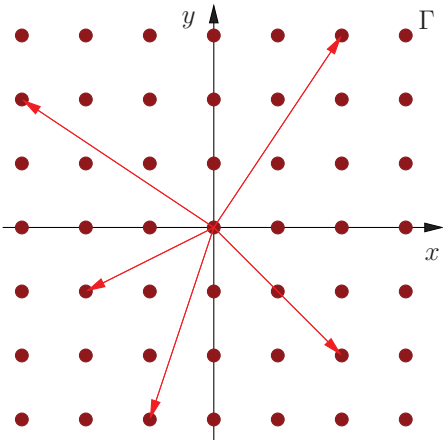

(c) Optic flow embedding

Figure 5. Different embeddings for a label space. In an ordered embedding, all labels are mapped onto a line, while for the Potts model, every label is mapped onto a different unit vector. For optical flow, each label is already a vector in $\mathbb{R}^{2}$, so a sensible embedding is given by the identity.

the $y$-direction, respectively. The Euclidean distance is a sensible distance on the components to regularize the flow field, corresponding to the regularizer of the TV- $L^{1}$ functional in [43]. Optic flow (and other geometric kinds of labels) would, however, more naturally be modeled with a continuous label space using one of the continuous regularizers in the later subsections.

5.2. New relaxation for the discrete label space. We will now show how to formulate the regularizer $J_{k}^{A}$ defined above in the new more general framework. While the previous formulation (5.3) already yields a relaxation to nonbinary functions $\boldsymbol{v}$, we will see that our framework results in a provably tighter one.

Taking a look at Theorem 5.1, we see that the regularizer must penalize the length of the jump set weighted by the label distance. Thus, our general regularizer in (3.12) must reduce to

$$
J_{k}\left(u_{k}\right)=\int_{S_{u_{k}}} d_{k}\left(u_{k}^{-}, u_{k}^{+}\right) \mathrm{d} \mathcal{H}^{n-1}
$$

where $d_{k}$ is the same metric as that used above in the representation (5.1). We can see that in order to reduce the general form to the one above, we must enforce a piecewise constant labeling, since the approximate gradient $\nabla u_{k}$ must be constantly zero outside the jump set. Applying Theorem 4.1, we can find a convex representation of $J_{k}$ in terms of the variables $\boldsymbol{v}$, which we formulate in the following proposition in its discretized form.

Proposition 5.2. A convex representation of (5.6) in terms of the variables $\boldsymbol{v}$ is given by

$$
J_{k}\left(u_{k}\right)=\sup _{\boldsymbol{p} \in C_{k}}\left\{\sum_{\lambda \in \Lambda_{k}} \int_{\Omega} v_{k}^{\lambda} \operatorname{div}\left(\boldsymbol{p}^{\lambda}\right) \mathrm{d} x\right\},
$$


with

$$
\begin{aligned}
C_{k}=\left\{\boldsymbol{p}: \Omega \times \Lambda_{k} \rightarrow \mathbb{R}^{n}:\right. & \boldsymbol{p}^{\lambda} \in C_{c}^{1}\left(\Omega ; \mathbb{R}^{n}\right) \text { for all } \lambda \in \Lambda_{k} \text { and } \\
& \left.\left|\boldsymbol{p}^{\lambda}-\boldsymbol{p}^{\mu}\right|_{2} \leq d_{k}(\lambda, \mu) \text { for all } \lambda, \mu \in \Lambda_{k}\right\} .
\end{aligned}
$$

We can now establish the relationship between our framework and the regularizer $J_{k}^{A}$ derived from a representation of the labels in (5.3) and show that ours is tighter.

Proposition 5.3. Let the regularizer $J_{k}$ be defined by the relaxation on the right-hand side in (5.7). Then for all $\boldsymbol{v}_{k} \in \mathcal{L}^{2}\left(\Omega, \operatorname{co}\left(\Delta_{k}\right)\right)$,

$$
J_{k}\left(\boldsymbol{v}_{k}\right) \geq J_{k}^{A}\left(\boldsymbol{v}_{k}\right) .
$$

Equality holds if $\boldsymbol{v}_{k}$ is binary.

The right-hand side of inequality (5.9) is exactly the previous regularizer used in [15, 22]. This implies that for binary functions, the regularizers coincide, which can already be seen from representation (5.6); see Theorem 5.1. However, if we perform the relaxation to functions taking values between 0 and 1, inequality (5.9) implies that the new relaxation is tighter, leading to solutions closer to the global optimum.

We will show in the remainder of the section that in addition to handling the discrete case better, our method also can handle continuous regularizers which penalize a smooth variation of the labels. This is not possible with the piecewise constant approach of $[22,15]$ which uses vectorial TV. For instance, our formulation is capable of representing more sophisticated regularizers such as Huber-TV and the piecewise smooth Mumford-Shah functional, as we will show in the following subsection. For the regularizers presented in the remainder of this section, relaxations have previously been proposed for the case of a one-dimensional label space in $[9,26,27,35]$. However, the framework presented here is more general and allows us to combine them freely in the different label dimensions.

5.3. Linear (TV) and truncated linear. For many applications, it is useful to penalize the difference between two label values $\lambda$ and $\mu$ only up to a certain threshold, reasoning that once they are that different, it no longer matters exactly how different they are. This means that if $|\lambda-\mu|$ becomes greater than a certain value $t$, jumps from $\lambda$ to $\mu$ are still penalized, but only by the constant $t$. Using linear penalization for small values, this leads to the robust truncated linear regularizer [9]

$$
J_{k}\left(u_{k}\right)=\int_{\Omega \backslash S_{u_{k}}}\left|\nabla u_{k}\right|_{2} \mathrm{~d} x+\int_{S_{u_{k}}} \min \left(t,\left|u_{k}^{+}-u_{k}^{-}\right|\right) \mathrm{d} \mathcal{H}^{n-1}(s) .
$$

The constraint set (4.4) for this case is

$$
\begin{gathered}
C_{k}=\left\{(\boldsymbol{p}, b) \in C_{c}^{1}\left(\Omega \times \Lambda_{k} ; \mathbb{R}^{n} \times \mathbb{R}\right) \text { such that for all } \lambda, \mu \in \Lambda_{k},\right. \\
\left.\left|\partial_{\lambda} \boldsymbol{p}^{\lambda}\right|_{2} \leq 1, \quad\left|\boldsymbol{p}^{\lambda}-\boldsymbol{p}^{\mu}\right|_{2} \leq t, \quad b=0\right\}
\end{gathered}
$$


The second constraint needs to be imposed only if $|\lambda-\mu| \geq t$, since otherwise it is already implied by the first constraint. In particular, the standard linear (TV) penalizer can be implemented by letting $t \rightarrow \infty$ and using only the first constraint.

5.4. Cyclic penalizer, TV- $S^{1}$. Some applications have a cyclic or circular set of labels, for example, regularization in the hue component in HSV or HSL color space. In this case, the distance between the last and first labels is the same as between any other subsequent pair of labels. This form of regularization was discussed in the functional lifting setting in the recent work [35] and can be expressed in our framework by setting

$$
J_{k}\left(u_{k}\right)=\int_{\Omega \backslash S_{u_{k}}}\left|\nabla u_{k}\right|_{2} \mathrm{~d} x+\int_{S_{u_{k}}} \min \left(u_{k}^{+}-u_{k}^{-}, 1-\left(u_{k}^{+}-u_{k}^{-}\right)\right) \mathrm{d} \mathcal{H}^{n-1}(s)
$$

for functions $u_{k}$ with range $\Lambda_{k}:=[0,1)$. The corresponding constraint set in its discretized form is given by

$$
\begin{gathered}
C_{k}=\left\{(\boldsymbol{p}, b) \in C_{c}^{1}\left(\Omega \times \Lambda_{k} ; \mathbb{R}^{n} \times \mathbb{R}\right) \text { such that for all } \lambda \in \Lambda_{k},\right. \\
\left.\left|\boldsymbol{p}^{\lambda}-\boldsymbol{p}^{\lambda+1}\right|_{2} \leq 1, \quad b \equiv 0\right\},
\end{gathered}
$$

where the circularly ordered label space $\Lambda_{k}$ is represented by integers $\left\{0, \ldots, N_{k}-1\right\}$ with addition modulo $N_{k}$.

5.5. Huber-TV. The TV regularization is known to produce staircasing effects in the reconstruction; i.e., the solution will be piecewise constant. While this is natural in the case of a discrete label space, for continuous label spaces it impedes smooth variations of the solution. A remedy for this is replacing the norm $\left|\nabla u_{k}\right|_{2}$ of the gradient by $h_{\alpha}\left(\nabla u_{k}\right)$ with the Huber function

$$
h_{\alpha}(z):= \begin{cases}\frac{1}{2 \alpha}|z|_{2}^{2} & \text { if }|z|_{2}<\alpha, \\ |z|_{2}-\frac{\alpha}{2} & \text { else }\end{cases}
$$

for some $\alpha>0$, which smooths out the kink at the origin. The Huber-TV regularizer is then defined by

$$
J_{k}\left(u_{k}\right)=\int_{\Omega} h_{\alpha}\left(\nabla u_{k}\right) \mathrm{d} x+\int_{S_{u_{k}}}\left|u_{k}^{+}-u_{k}^{-}\right| \mathrm{d} \mathcal{H}^{n-1}(s) .
$$

The limiting case $\alpha=0$ leads to the usual TV regularization. Theorem (4.1) gives a convex representation for $J_{k}$; see also [27]. The constraint set in (4.4) is found to be

$$
\begin{gathered}
C_{k}=\left\{(\boldsymbol{p}, b) \in C_{c}^{1}\left(\Omega \times \Lambda_{k} ; \mathbb{R}^{n} \times \mathbb{R}\right) \text { such that for all } \lambda \in \Lambda_{k},\right. \\
\left.b^{\lambda} \geq \frac{\alpha}{2}\left|\partial_{\lambda} \boldsymbol{p}^{\lambda}\right|_{2}^{2}, \quad\left|\partial_{\lambda} \boldsymbol{p}^{\lambda}\right|_{2} \leq 1\right\} .
\end{gathered}
$$


5.6. Piecewise smooth Mumford-Shah model. The celebrated Mumford-Shah regularizer $[2,26]$

$$
J_{k}\left(u_{k}\right)=\int_{\Omega \backslash S_{u_{k}}} \frac{1}{2 \alpha}\left|\nabla u_{k}\right|_{2}^{2} \mathrm{~d} x+\nu \mathcal{H}^{n-1}\left(S_{u_{k}}\right)
$$

with parameters $\alpha, \nu>0$ allows us to estimate a denoised image $u_{k}$ which is piecewise smooth. Parameter $\nu$ can be used to easily control the total length of the jump set $S_{u_{k}}$. Bigger values of $\nu$ lead to a smaller jump set; i.e., the solution will be smooth on wider subregions of $\Omega$. The constraint set in the convex representation of Theorem 4.1 becomes

$$
\begin{gathered}
C_{k}=\left\{(\boldsymbol{p}, b) \in C_{c}^{1}\left(\Omega \times \Lambda_{k} ; \mathbb{R}^{n} \times \mathbb{R}\right) \text { such that for all } \lambda, \mu \in \Lambda_{k}\right. \\
\left.b^{\lambda} \geq \frac{\alpha}{2}\left|\partial_{\lambda} \boldsymbol{p}^{\lambda}\right|_{2}^{2}, \quad\left|\boldsymbol{p}^{\lambda}-\boldsymbol{p}^{\mu}\right|_{2} \leq \nu\right\}
\end{gathered}
$$

The limiting case $\alpha=0$ gives the piecewise constant Mumford-Shah regularizer (also called Potts regularizer), which can also be obtained from Proposition 5.2 by setting $d_{k}(\lambda, \mu)=\nu$ for all $\lambda \neq \mu$. Compared to (5.3), this alternative yields a tighter but more memory intensive relaxation for the Potts regularizer $[9,25]$.

\section{Implementation.}

6.1. Final relaxation to a convex problem. In order to transform the multilabel problem into the final form which we are going to solve, we formulate it in terms of the indicator functions $v_{k}^{\lambda}$ on the discretized label space using the representation (4.3) for the regularizer and the relaxation (4.10) of the data term. Discretization of the label space is necessary now to arrive at a well-posed problem. Let us briefly summarize and review the objects we are dealing with in the final problem. The minimizer we are looking for is a vector $\boldsymbol{v}=\left(\boldsymbol{v}_{k}\right)_{k=1 . . d}$ of functions $\boldsymbol{v}_{k} \in \mathcal{L}^{2}\left(\Omega, \operatorname{co}\left(\Delta_{k}\right)\right)$, which means that we are looking for a minimizer in a convex set $\mathcal{D}$,

$$
\begin{aligned}
\boldsymbol{v} \in \mathcal{D}:=\left\{\boldsymbol{v} \in \mathcal{L}^{2}\left(\Omega, \mathbb{R}^{N_{1}+\cdots+N_{d}}\right)\right. & \text { such that for all } x \in \Omega, \boldsymbol{v}(x) \in \operatorname{co}\left(\Delta_{\times}\right), \\
& \text {with } \left.\Delta_{\times}=\Delta_{1} \times \cdots \times \Delta_{d}\right\} .
\end{aligned}
$$

In the convex hull co $\left(\Delta_{\times}\right)=\operatorname{co}\left(\Delta_{1}\right) \times \cdots \times \operatorname{co}\left(\Delta_{d}\right)$ each co $\left(\Delta_{k}\right)$ is given by the same expression as in (3.3) but with the set $\{0,1\}$ replaced by $[0,1]$.

Let us now turn to the regularizer, which is defined via the relaxation in Theorem 4.1. The key ingredients are the convex sets $C_{k}$ which depend on the kind of regularization we want to use possible options were detailed in the last section. Let $\mathcal{C}:=C_{1} \times \cdots \times C_{d}$ denote the convex set of all regularizer dual variables, and define the linear operator $K: \mathcal{C} \rightarrow$ $\mathcal{L}^{2}\left(\Omega, \mathbb{R}^{N_{1}+\cdots+N_{d}}\right)$ via

$$
K(\boldsymbol{p}, \boldsymbol{b}):=\left(-\operatorname{div}\left(\boldsymbol{p}_{k}^{\lambda}\right)-b_{k}^{\lambda}\right)_{k=1, \ldots, d, \lambda \in \Lambda_{k}}
$$


Then Theorem 4.1 in fact shows that the regularizer can be written in terms of $\boldsymbol{v}$ in the simple form

$$
J(\boldsymbol{v})=\sup _{(\boldsymbol{p}, \boldsymbol{b}) \in \mathcal{C}}\{\langle K(\boldsymbol{p}, \boldsymbol{b}), \boldsymbol{v}\rangle\}
$$

where $\langle\cdot, \cdot\rangle$ denotes the inner product on $\mathcal{L}^{2}\left(\Omega, \mathbb{R}^{N_{1}+\cdots+N_{d}}\right)$. The fully relaxed problem we are going to solve can now be written as

$$
\underset{\boldsymbol{v} \in \mathcal{D}}{\operatorname{argmin}}\left\{J(\boldsymbol{v})+R_{\text {data }}(\boldsymbol{v})\right\}
$$

using the relaxation $R_{\text {data }}$ of the data term defined in (4.10). It is straightforward to prove the existence of solutions.

Proposition 6.1. Problem (6.4) always has a minimizer $\hat{\boldsymbol{v}} \in \mathcal{D}$.

Note that because of the relaxation, the solution might not be binary. If it already has values in $\Delta_{k}$, we have found the global optimum of the original problem (1.1); otherwise we have to project the result back to the smaller set of binary-valued functions. For this, let $\hat{\boldsymbol{v}}$ be a minimizer of the final relaxation (6.4). Thus, the functions $\hat{v}_{k}^{\lambda}$ might have values between 0 and 1. In order to obtain a feasible solution to the original problem (1.1), we just project back to the space of allowed functions. The function $\hat{\boldsymbol{u}} \in \mathcal{L}^{2}(\Omega, \Gamma)$ closest to $\hat{\boldsymbol{v}}$ is given by setting

$$
\hat{\boldsymbol{u}}(x)=\underset{\gamma \in \Gamma}{\operatorname{argmax}}\left\{\hat{v}_{1}^{\gamma_{1}}(x) \times \cdots \times \hat{v}_{d}^{\gamma_{d}}(x)\right\}
$$

i.e., we choose the label where the combined indicator functions have the highest value. This is the same as choosing the label by maximizing each component $v_{k}$ separately:

$$
\hat{\boldsymbol{u}}(x)=\gamma \quad \text { with } \gamma_{k}=\underset{\lambda \in \Lambda_{k}}{\operatorname{argmax}}\left\{\hat{v}_{k}^{\lambda}(x)\right\} \quad \text { for all } 1 \leq k \leq d .
$$

We cannot guarantee that the solution $\hat{\boldsymbol{u}}$ is indeed a global optimum of the original problem (1.1), since there is nothing equivalent to the thresholding theorem [11] known for this kind of relaxation. However, we still can give a bound for how close we are to the global optimum. Indeed, the energy of the optimal solution of (1.1) must lie somewhere between the energies of $\hat{\boldsymbol{v}}$ and $\hat{\boldsymbol{u}}$, as previously explained in the beginning of section 4 .

6.2. Numerical method. Using the representation (6.3) for $J$, and the definition (4.10) for the relaxation $R_{\text {data }}$, we can transform the final formulation (6.4) of the multilabel problem into the saddle point problem

$$
\min _{\boldsymbol{v} \in \mathcal{D}} \max _{\substack{(\boldsymbol{p}, \boldsymbol{b}) \in \mathcal{C} \\ \boldsymbol{q} \in \mathcal{Q}}}\{\langle K(\boldsymbol{p}, \boldsymbol{b})+\boldsymbol{q}, \boldsymbol{v}\rangle\}
$$

We minimize the energy (6.7) with a recent general fast primal-dual algorithm from [10], which is designed for this type of problem. The algorithm is essentially a gradient descent in the primal variable $\boldsymbol{v}$ and a gradient ascent in the dual variables $\boldsymbol{p}, \boldsymbol{b}$, and $\boldsymbol{q}$, with a subsequent 
application of proximation operators, which act as generalized reprojections. In our case, these are just the usual orthogonal projections onto the respective constraint sets $\mathcal{D}, \mathcal{C}$, and $\mathcal{Q}$. The algorithm update equations for our case can be derived in a straightforward manner from the general Algorithm 1 presented in [10]. The remaining question is therefore only how to compute the projections onto the constraint sets after each algorithm iteration. Since these sets are defined by numerous nonlocal constraints, a direct projection is quite costly.

Therefore, we suggest implementing as many constraints as possible using Lagrange multipliers by adding specific additional terms to the energy. This comes at the cost of having more terms in the final overall energy and that the optimization is also done over additional variables, the Lagrange multipliers. However, in the end, fewer of the explicit constraints remain, so that the projections become easier to calculate. Also, the algorithm complexity remains the same since the update equations are still straightforward.

First, the simplex constraint $\boldsymbol{v} \in \mathcal{D}$, i.e., $v_{k} \in \operatorname{co}\left(\Delta_{k}\right)$ with $\Delta_{k}$ in (3.3) for $1 \leq k \leq d$, is enforced by adding the Lagrange multiplier terms

$$
\sup _{\sigma} \sum_{k=1}^{d} \int_{\Omega} \sigma_{k}(x)\left(\sum_{\lambda \in \Lambda_{k}} v_{k}^{\lambda}(x)-1\right) \mathrm{d} x
$$

to the energy (6.7), optimizing over $\sigma: \Omega \rightarrow \mathbb{R}^{d}$ in addition to the other variables. This leaves just the simple condition $\boldsymbol{v} \geq 0$ for the indicator variables $\boldsymbol{v}$. We note that it is also possible to implement the simplex constraint explicitly by the iterative algorithm [23]. However, in the end this increases the computation time per iteration many times over since the projection then requires $\mathcal{O}\left(N_{1}+\cdots+N_{d}\right)$ steps in the worst case. Also, the explicit projection reduces only slightly the number of iterations needed to compute a minimizer of (6.7) to a certain precision. Therefore, overall the Lagrange multiplier approach turns out to be faster and is also easier to implement.

Next, we enforce the constraints $(\boldsymbol{p}, \boldsymbol{b}) \in \mathcal{C}$ on the dual variables of the regularizer by introducing new variables

$$
\boldsymbol{d}_{k}^{\lambda}=\partial_{\lambda} \boldsymbol{p}_{k}^{\lambda} \quad \text { or } \quad \boldsymbol{d}_{k}^{\lambda \mu}=\boldsymbol{p}_{k}^{\lambda}-\boldsymbol{p}_{k}^{\mu}
$$

depending on the kind of constraints in $C_{k}$. To enforce these equalities, we add the corresponding Lagrange multiplier terms

$$
\inf _{\boldsymbol{\eta}} \int_{\Omega} \boldsymbol{\eta}_{k}^{\lambda}\left(\partial_{\gamma} \boldsymbol{p}_{k}^{\lambda}-\boldsymbol{d}_{k}^{\lambda}\right) \mathrm{d} x \quad \text { or } \quad \inf _{\boldsymbol{\eta}} \int_{\Omega} \boldsymbol{\eta}_{k}^{\lambda, \mu}\left(\boldsymbol{p}_{k}^{\lambda}-\boldsymbol{p}_{k}^{\mu}-\boldsymbol{d}_{k}^{\lambda \mu}\right) \mathrm{d} x
$$

for each $1 \leq k \leq d$ and $\lambda \in \Gamma_{k}$, respectively, $\lambda, \mu \in \Gamma_{k}$, to the energy. Instead of computing the projection of $(\boldsymbol{p}, \boldsymbol{b})$ in each step, we can then perform the projection of the new variables $\left(\boldsymbol{d}_{k}, \boldsymbol{b}_{k}\right)$ on a corresponding constraint set. The advantage is that the overall projection decouples into independent projections of $\boldsymbol{d}_{k}^{\lambda}$ or $\boldsymbol{d}_{k}^{\lambda \mu}$ and $b_{k}^{\lambda}$ onto simple convex sets, which are easy to implement. Alternatively, constraints of the form $\left|\boldsymbol{p}_{k}^{\lambda}-\boldsymbol{p}_{k}^{\mu}\right|_{2} \leq m$ as used in (5.8), (5.11), and (5.18) can be enforced using convex duality, by adding the terms

$$
\inf _{\boldsymbol{\eta}} \int_{\Omega} \boldsymbol{\eta}_{k}^{\lambda, \mu}\left(\boldsymbol{p}_{k}^{\lambda}-\boldsymbol{p}_{k}^{\mu}\right)+m\left|\boldsymbol{\eta}_{k}^{\lambda, \mu}\right|_{2} \mathrm{~d} x
$$


Table 1

The table shows the total amount of memory required for the implementations of the previous and proposed methods depending on the size of the problem (using TV regularization). For the proposed method, the projection (6.12) of the data term dual variables can be implemented either globally (g), or more slowly but more memory efficient as a subproblem of the proximation operator $(\mathrm{p})$, here using $N_{1} / 5$ chunks. Also shown is the total run time for 5000 iterations, which usually suffices for convergence. Numbers in red indicate a memory requirement larger than what fits on the largest currently available CUDA capable devices (6 GiB). Note that the proposed framework can still handle all problem sizes above.

\begin{tabular}{|c|c||c|c||c|c|}
\hline \# Pixels & \# Labels & \multicolumn{2}{|c||}{ Memory $(\mathrm{MiB})$} & \multicolumn{2}{c|}{ Run time $(\mathrm{s})$} \\
$P=P_{x} \times P_{y}$ & $N_{1} \times N_{2}$ & previous & proposed $(\mathrm{g} / \mathrm{p})$ & previous & proposed $(\mathrm{g} / \mathrm{p})$ \\
\hline $320 \times 240$ & $8 \times 8$ & 112 & $112 / 102$ & 31 & $26 / 140$ \\
$320 \times 240$ & $16 \times 16$ & 450 & $337 / 168$ & 125 & $80 / 488$ \\
$320 \times 240$ & $32 \times 32$ & 1800 & $1124 / 330$ & 503 & $215 / 1953$ \\
$320 \times 240$ & $50 \times 50$ & 4394 & $2548 / 504$ & 2110 & $950 / 5188$ \\
$320 \times 240$ & $64 \times 64$ & 7200 & $4050 / 657$ & - & $1100 / 8090$ \\
\hline $640 \times 480$ & $8 \times 8$ & 448 & $521 / 413$ & 127 & $102 / 560$ \\
$640 \times 480$ & $16 \times 16$ & 1800 & $1351 / 676$ & 539 & $295 / 1945$ \\
$640 \times 480$ & $32 \times 32$ & 7200 & $4502 / 1327$ & - & $1290 / 7795$ \\
$640 \times 480$ & $50 \times 50$ & 17578 & $10197 / 2017$ & - & $-/ 32887$ \\
$640 \times 480$ & $64 \times 64$ & 28800 & $16202 / 2627$ & - & $-/ 48583$ \\
\hline
\end{tabular}

to the energy instead of (6.10). We used this way in our implementation, as it turns out to be much faster in practice. The optimization (6.7) is now performed over the primals $\boldsymbol{v}, \boldsymbol{d}, \boldsymbol{\eta}$ and the duals $\boldsymbol{p}, \boldsymbol{b}, \boldsymbol{q}, \boldsymbol{\sigma}$.

Finally, the projection of a $\tilde{\boldsymbol{q}}$ onto $\mathcal{Q}$ consists of solving

$$
\underset{\boldsymbol{q} \in \mathcal{Q}}{\operatorname{argmin}}\left\{\sum_{k=1}^{d} \sum_{\lambda \in \Lambda_{k}}\left(q_{k}^{\lambda}-\tilde{q}_{k}^{\lambda}\right)^{2}\right\}
$$

pointwise for each $x \in \Omega$. The number of constraints in $\mathcal{Q}$, as defined in (4.11), equals the total number of labels in the product space $\Gamma=\Lambda_{1} \times \cdots \times \Lambda_{d}$. Unfortunately, implementing these constraints by adding Lagrange multiplier terms

$$
\inf _{\boldsymbol{\mu} \geq 0}-\int_{\Omega} \sum_{\gamma \in \Gamma} \mu^{\gamma}(x)\left(q_{1}^{\gamma_{1}}(x)+\cdots+q_{d}^{\gamma_{d}}(x)-c^{\gamma}(x)\right) \mathrm{d} x
$$

to the global problem (6.7), i.e., for each $x \in \Omega$, is not possible for larger problems since it requires too many additional variables $\boldsymbol{\mu}$ to be memory efficient.

Thus, for larger problems, the projection needs to be computed explicitly after each outer iteration as a subproblem by solving (6.12), which increases the run time; see Table 1. To make sure that $\boldsymbol{q}$ lies in $\mathcal{Q}$, we add the corresponding Lagrange multiplier terms to the local energy (6.12). This results in another saddle point problem to be optimized over now unconstrained $\boldsymbol{q}$ and $\boldsymbol{\mu} \geq 0$, which we again solve using the algorithm in [10]. Specifically, since the $\boldsymbol{q}$-only terms are uniformly convex, we use Algorithm 2 of [10] with the accelerated $\mathcal{O}\left(\frac{1}{N_{\text {iter }}^{2}}\right)$ convergence rate. Since there is only a small change in the variables $\boldsymbol{q}$ per outer iteration, only a small number of inner iterations is required. In our experiments, we used 10 inner iterations. 
6.3. Memory requirements. When the domain $\Omega$ is discretized into $P$ pixels, the primal and dual variables are represented as matrices. There are essentialy two types of data. The first type is relatively cheap to store, since memory requirements scale with the sum $N_{1}+$ $\cdots+N_{d}$ of the independent dimensions. The second type is expensive, since it scales with $N_{1} \cdots N_{d}$. The variables and constants appearing in the energy are classified in the following table, where $n=\operatorname{dim} \Omega$ is the dimension of the image domain $\Omega$.

\begin{tabular}{|l|l|}
\hline Variable or constant & Floating point numbers \\
\hline $\boldsymbol{\sigma}$ & $P \cdot d$ \\
$\boldsymbol{v}, \boldsymbol{q}$ & $P \cdot\left(N_{1}+\cdots+N_{d}\right)$ \\
$\boldsymbol{p}$ & $n P \cdot\left(N_{1}+\cdots+N_{d}\right)$ \\
$\boldsymbol{\mu}, \boldsymbol{c}$ & $P \cdot\left(N_{1} \cdots N_{d}\right)$ \\
\hline
\end{tabular}

The relaxation of the regularizer incurs additional costs per label space dimension, depending on the type of regularizer. This is summarized in the following table.

\begin{tabular}{|l|l|l|}
\hline Regularizer & Additional variables & Additional floating point numbers \\
\hline Potts using (5.3) & - & - \\
TV or cyclic TV & $\boldsymbol{\eta}_{k}^{\lambda}, \boldsymbol{d}_{k}^{\lambda}$ & $2 n P \cdot N_{k}$ \\
Huber-TV & $\boldsymbol{\eta}_{k}^{\lambda}, \boldsymbol{d}_{k}^{\lambda}, b_{k}^{\lambda}$ & $(2 n+1) P \cdot N_{k}$ \\
Truncated linear & $\boldsymbol{\eta}_{k}^{\lambda, \mu}$ & $n P \cdot N_{k}\left(N_{k}-1\right) / 2$ \\
Potts using (5.18), $\alpha=0$ & $\boldsymbol{\eta}_{k}^{\lambda, \mu}$ & $n P \cdot N_{k}\left(N_{k}-1\right) / 2$ \\
Piecewise smooth & $\boldsymbol{\eta}_{k}^{\lambda, \mu}, \boldsymbol{\eta}_{k}^{\lambda}, \boldsymbol{d}_{k}^{\lambda}, b_{k}^{\lambda}$ & $n P \cdot N_{k}\left(N_{k}-1\right) / 2+(2 n+1) P \cdot N_{k}$ \\
\hline
\end{tabular}

Obviously, truncated linear and piecewise smooth regularization can require a lot of additional memory if the dimensions of the factors are large. They seem therefore practically feasible only when the label space consists of many small factors. However, the projections onto the regularizer constraints can also be solved locally in each iteration for each dimension separately. This removes the need to store these variables globally at the cost of additional computation time.

The most expensive variable is $\boldsymbol{\mu}$, appearing in the global problem (6.13) or local problem (6.12), respectively. If we have enough memory to store it, it is more efficient to solve the global problem. However, it is also possible here to trade computation time for a reduction in memory requirements by solving the local problem (6.12) in each iteration. For this, note that (6.12) can be separated into independent subproblems for each $x \in \Omega$ and can be solved in chunks of points $x$ in parallel. The size of the chunks can be chosen to fit into the available memory; ideally we choose it as large as possible for maximum parallelization.

Finally, note that the data term $\boldsymbol{c}$ is an expensive constant to store. If there is not enough graphics processing unit (GPU) memory for it, it is possible, e.g., to hold it in main memory and transfer separate layers of it during computation of the primal prox operator to the GPU. This increases computation time by a factor of $5-10$, so it is usually much more efficient to compute the data term on the fly on the GPU if it is of a simple form. 
In summary, with the above reduction techniques it is possible to get rid of all memory expensive variables and constants at the cost of more computation time. To give an idea about the final requirements, they are compared for the case of a two-dimensional label space and TV penalization in Table 1 . Note that the memory requirements for the original method without using the reduction are $(n+2) P \cdot\left(N_{1} \cdots N_{d}\right)$ to store all primal and dual variables even for the simplest regularizer, while all regularizer costs scale with $N=N_{1} \cdots N_{d}$ according to the second table on the previous page. Statistics for a three-dimensional label space with different regularizers can be found in Table 2. Clearly, large scale problems can be solved only by using the proposed reduction technique.

7. Experiments. We demonstrate the correctness and usability of our method on several examples. Different regularizers are used in the examples. In the cases where the regularizer can be simulated with the previous relaxation [15], we compared the resulting optimality bounds. On average, our bounds were approximately three times better (3-5\% with the proposed framework compared to $10-15 \%$ with the previous relaxation). All experiments were performed with a parallel CUDA implementation running on a NVIDIA GTX 680 GPU for section 7.1, and on a TESLA C2070 for all other experiments. The time steps for the algorithm are chosen automatically using the recent preconditioned version [24] of [10]. The number of iterations in each experiment is chosen appropriately so that visually the solution remains stable and does not change anymore (usually 1000-5000 depending on problem size).

7.1. Segmentation. Multidimensional label spaces occur naturally in the problem of image segmentation, where a multichannel input image $f$ is segmented according to a local cost equal to the squared distance to the labels,

$$
c^{\gamma}(x)=\sum_{k=1}^{d}\left(f_{k}(x)-\gamma_{k}\right)^{2} .
$$

Typical multichannel images are, of course, color images with various color spaces which are usually three-dimensional; see Figure 6 for some segmentation examples.

We choose this archetypical problem for an extensive comparison of our method to different labeling approaches. We first compare our proposed scheme for vectorial multilabel problems (VML) to the similar continuous method for a scalar label space (SML) [22]. For comparisons with discrete approaches, we used the MRF software accompanying the comparative study in [38]. ${ }^{2}$ We compare to the $\alpha$-expansion $(\alpha$-EXP) and $\alpha$ - $\beta$-swap (SWAP) algorithms based on the maxflow library $[6,5,21]$ using the newest updated implementation for [13]. ${ }^{3}$ We also compare to two message-passing methods, max-product belief propagation (BP) [39] and sequential tree-reweighted message passing (TRW-S) [40, 19].

Table 2 shows detailed statistics of memory requirements, run time, and error bounds. Throughout all experiments, we used a fixed number of iterations to keep results comparable, tuned to be sufficient for convergence on intermediate-sized label spaces. For VML and SML, we used 1000 iterations; for TRW-S and BP, 50 iterations; and for $\alpha$-EXP and SWAP, 5 iterations.

\footnotetext{
${ }^{2}$ For MRF 2.1, see http://vision.middlebury.edu/MRF/code/.

${ }^{3}$ For the gco-v3 library, see http://vision.csd.uwo.ca/code/.
} 
Table 2

Performance comparison of several continuous and discrete multilabel algorithms on the segmentation problem using a three-dimensional label space and different regularizers. Results are averaged over 10 different images with average resolution of 0.7 megapixels. See section 7.1 for a discussion.

Potts regularizer (using (5.3) for the continuous methods)

\begin{tabular}{|l||ccc|ccc|}
\hline \multirow{2}{*}{ Algorithm } & mem $(\mathrm{MiB})^{1}$ & $\begin{array}{c}4 \times 4 \times 4 \\
\text { time }(\mathrm{s})^{2}\end{array}$ & bound $(\%)^{3}$ & mem $(\mathrm{MiB})^{1}$ & $\begin{array}{c}6 \times 6 \times 6 \\
\text { time }(\mathrm{s})^{2}\end{array}$ & bound $(\%)^{3}$ \\
\hline VML & 367 & 15.25 & 0.46 & 998 & 42.83 & 0.72 \\
SML & 607 & 16.81 & 1.43 & $>4 \mathrm{GiB}$ & - & - \\
$\alpha$-EXP & 677 & 25.13 & $0.20^{4}$ & 1369 & 88.90 & $0.54^{4}$ \\
SWAP & 677 & 29.84 & $0.28^{5}$ & 1369 & 137.57 & $0.81^{5}$ \\
BP & 1368 & 36.63 & $6.94^{5}$ & 4256 & 119.43 & $11.56^{5}$ \\
TRW-S & 1368 & 40.06 & 0.16 & 4256 & 129.52 & 0.80 \\
\hline & & $8 \times 8 \times 8$ & & \multicolumn{3}{c}{$10 \times 10 \times 10$} \\
& mem $(\mathrm{MiB})^{1}$ & time $(\mathrm{s})^{2}$ & bound $(\%)^{3}$ & mem $(\mathrm{MiB})^{1}$ & time $(\mathrm{s})^{2}$ & bound $(\%)^{3}$ \\
\hline VML & 2173 & 94.59 & 1.03 & $2185^{6}$ & $209.34^{6}$ & 0.80 \\
$\alpha$-EXP & 2746 & 173.64 & $0.90^{4}$ & 5020 & 343.67 & $1.01^{4}$ \\
SWAP & 2746 & 461.48 & $1.34^{5}$ & 5020 & 1472.45 & $1.62^{5}$ \\
BP & 8667 & 254.08 & $16.29^{5}$ & 16610 & 496.12 & $17.73^{5}$ \\
TRW-S & 8667 & 287.30 & 1.95 & 16610 & 539.51 & 2.70 \\
\hline
\end{tabular}

\begin{tabular}{|l||ccc|ccc|}
\multicolumn{7}{|c|}{ Linear (TV) regularizer } \\
Algorithm & mem $(\mathrm{MiB})^{1}$ & $\begin{array}{c}4 \times 4 \times 4 \\
\text { time }(\mathrm{s})^{2}\end{array}$ & bound $(\%)^{3}$ & mem $(\mathrm{MiB})^{1}$ & $\begin{array}{c}6 \times 6 \times 6 \\
\text { time }(\mathrm{s})^{2}\end{array}$ & bound $(\%)^{3}$ \\
\hline VML & 401 & 18.67 & 1.50 & 1055 & 48.09 & 1.67 \\
$\alpha$-EXP & 677 & 18.04 & $0.06^{4}$ & 1369 & 82.70 & -4 \\
SWAP & 677 & 23.67 & $0.16^{5}$ & 1369 & 139.90 & -5 \\
BP & $51000^{8}$ & $740.49^{8}$ & $5.87^{5}$ & $>64 \mathrm{GiB}$ & - & - \\
TRW-S & $51000^{8}$ & $746.00^{8}$ & 0.04 & $>64 \mathrm{GiB}$ & - & - \\
\hline & & $8 \times 8 \times 8$ & & \multicolumn{3}{c|}{$10 \times 10 \times 10$} \\
& mem $(\mathrm{MiB})^{1}$ & time $(\mathrm{s})^{2}$ & bound $(\%)^{3}$ & mem $(\mathrm{MiB})^{1}$ & time $(\mathrm{s})^{2}$ & bound $(\%)^{3}$ \\
\hline VML & 2253 & 101.75 & 2.27 & $2287^{6}$ & $217.66^{6}$ & 3.10 \\
$\alpha$-EXP & 2746 & 201.05 & -4 & 5020 & 408.93 & -4 \\
SWAP & 2746 & 504.47 & -5 & 5020 & 1576.32 & -5 \\
\hline
\end{tabular}

Truncated linear regularizer

\begin{tabular}{|c|c|c|c|c|c|c|}
\hline Algorithm & mem $(\mathrm{MiB})^{1}$ & $\begin{array}{l}4 \times 4 \times 4 \\
\text { time }(\mathrm{s})^{2}\end{array}$ & bound $(\%)^{3}$ & mem $(\mathrm{MiB})^{1}$ & $\begin{array}{c}6 \times 6 \times 6 \\
\text { time }(\mathrm{s})^{2}\end{array}$ & bound $(\%)^{3}$ \\
\hline$\overline{\text { VML }}$ & 435 & 20.77 & 0.95 & 1168 & 55.01 & 1.83 \\
\hline$\alpha$-EXP & 677 & 18.13 & $0.09^{4}$ & 1369 & 82.78 & -4 \\
\hline SWAP & 677 & 23.68 & $0.18^{5}$ & 1369 & 139.30 & $-^{5}$ \\
\hline $\mathrm{BP}$ & $51000^{8}$ & $750.36^{8}$ & $4.49^{5}$ & $>64 \mathrm{GiB}$ & - & - \\
\hline \multirow[t]{2}{*}{ TRW-S } & $51000^{8}$ & $741.80^{8}$ & 0.04 & $>64 \mathrm{GiB}$ & - & - \\
\hline & mem $(\mathrm{MiB})^{1}$ & $\begin{array}{l}8 \times 8 \times 8 \\
\text { time }(\mathrm{s})^{2}\end{array}$ & bound $(\%)^{3}$ & mem $(\mathrm{MiB})^{1}$ & $\begin{array}{c}0 \times 10 \times 10 \\
\text { time }(\mathrm{s})^{2}\end{array}$ & bound $(\%)^{3}$ \\
\hline VML & $1523^{6}$ & $132.33^{6}$ & 3.24 & $805^{6,7}$ & $1304^{6,7}$ & 3.50 \\
\hline$\alpha$-EXP & 2746 & 200.11 & -4 & 5020 & 408.73 & 4 \\
\hline SWAP & 2746 & 507 & $-^{5}$ & 5020 & 1578.29 & -5 \\
\hline
\end{tabular}

1: GPU memory for VML and SML, otherwise CPU memory (measured with valgrind memory profiler). Intel Core i7-3820 CPU at $3.8 \mathrm{GHz}$ with $64 \mathrm{GiB}$ of RAM, nVidia GTX $680 \mathrm{GPU}$ with $4 \mathrm{GiB}$ of RAM. Optimality bound in percent of the lower bound to solution provided by the algorithm.

No lower bound provided by algorithm, a theoretical (far from tight) a priori bound is known to be $\geq 100 \%[6,13]$. Bound was computed with lower bound returned by TRW-S, if any is given.

No lower bound provided by algorithm, no theoretical bound known as far as we know. See also 4.

No lower bound provided by algorithm, no theoretical bound known as far as we
Data term computed on the fly (saves GPU storage, minimal run-time increase).

6: Data term computed on the fly (saves GPU storage, minimal run-time increase).
Data term relaxation reprojection computed in each iteration reduces GPU memory usage by 2 GiB, but Data term relaxation reprojection computed
increases computation time by a factor of 5 .

increases computation time by a factor of 5 .
Requires general regularizer implementation, which is inefficient according to the author of the code. Unfortunately, no specialized implementation is available for three-dimensional regularizers. 


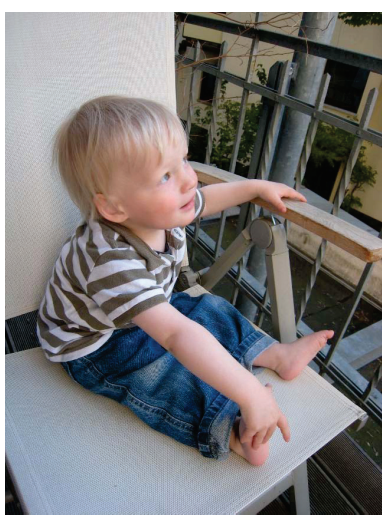

Input

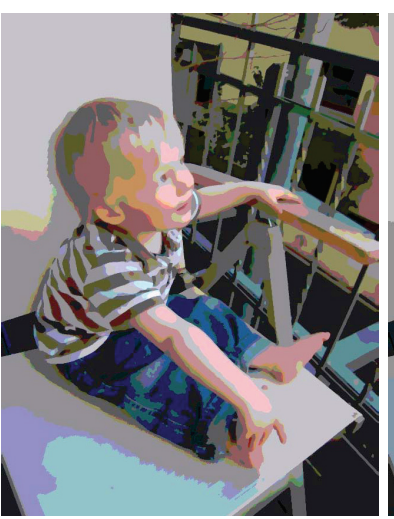

RGB $6 \times 6 \times 6$

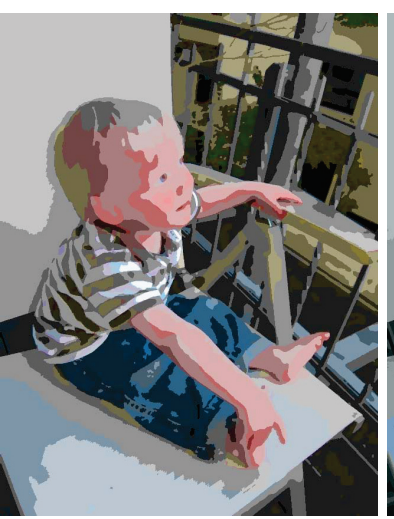

HSL $8 \times 5 \times 5$

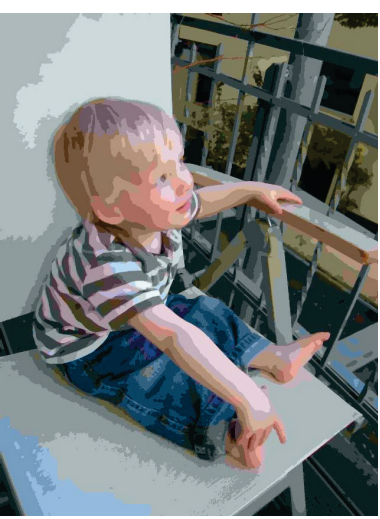

$\mathrm{L}^{*} \mathrm{a}^{*} \mathrm{~b}^{*} 8 \times 5 \times 5$

Figure 6. Segmentation using different three-dimensional spaces of equidistant color labels. The perceptually uniform color space CIELAB gives the visually most compelling results with the most natural looking color reproduction. There is a linear penalizer in all channels, except for the $H$ channel, which requires cyclic regularization. The above examples require less than two minutes run time, and all results are within $3 \%$ of the global optimum.

Memory requirements and largest problem size. Our method can deal with much larger problems than any of the other algorithms. On a high-end workstation with $64 \mathrm{GiB}$ of main memory, the generic implementation of TRW-S already stops working at $12^{3}$ labels for the Potts model. However, the implementation is extremely general, and requirements could probably be reduced by more specialized code. About the largest problem which can be solved with $\alpha$-EXP has $15^{3}$ labels, after which the reference implementation produces a segmentation fault - this hints at a hidden limitation of the implementation; memorywise it should be able to cope with about $17^{3}$.

In contrast, the GPU has only $4 \mathrm{GiB}$ of memory, and our method can still handle problems up to $31^{3}$ (almost 30000 labels), albeit with high run-time requirements. The table below shows limit cases at an image resolution of $640 \times 950$ - note that GPU memory shown is the theoretical minimum amount required, but we leverage all the remaining GPU memory to minimize the number of chunks for the local projections.

\begin{tabular}{|c|cc|cc|}
\hline & \multicolumn{2}{|c|}{ VML } & \multicolumn{2}{c|}{$\alpha$-EXP } \\
Label space & GPU mem $(\mathrm{MiB})$ & run time $(\min )$ & CPU mem $(\mathrm{MiB})$ & run time $(\min )$ \\
\hline $15 \times 15 \times 15$ & 550 & 79 & 18120 & 31 \\
$23 \times 23 \times 23$ & 836 & 485 & $>64 \mathrm{GiB}$ & (est. 480) \\
$31 \times 31 \times 31$ & 1123 & 1179 & $>64 \mathrm{GiB}$ & (est. 7680) \\
\hline
\end{tabular}

Performance. For smaller problems when we can use the global implementation of the constraints, our method outperforms the others in terms of run time, while attaining comparable optimality bounds. When the problems become larger, we need to switch to local projections per iteration, which increases run time fivefold and makes the other methods faster across a certain range of problem sizes. However, note that the run time of our algorithm scales better, so that at problem size $23^{3}$ the algorithm is about to break even with the estimated computation times of $\alpha$-EXP again, the latter approximately doubling its computation time every time the dimension of each factor is increased by 2 ; see Table 2 . 


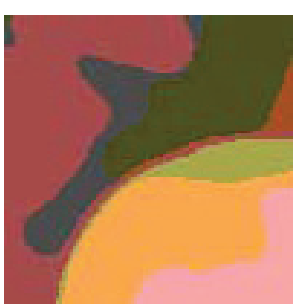

VML

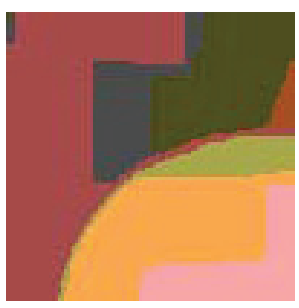

EXP

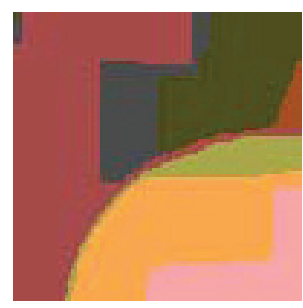

SWAP

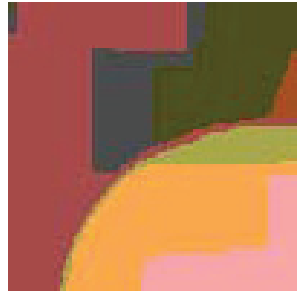

BP

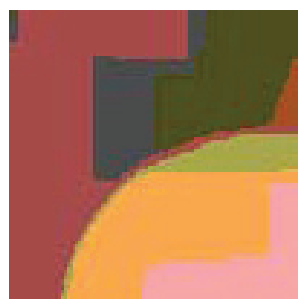

TRW-S

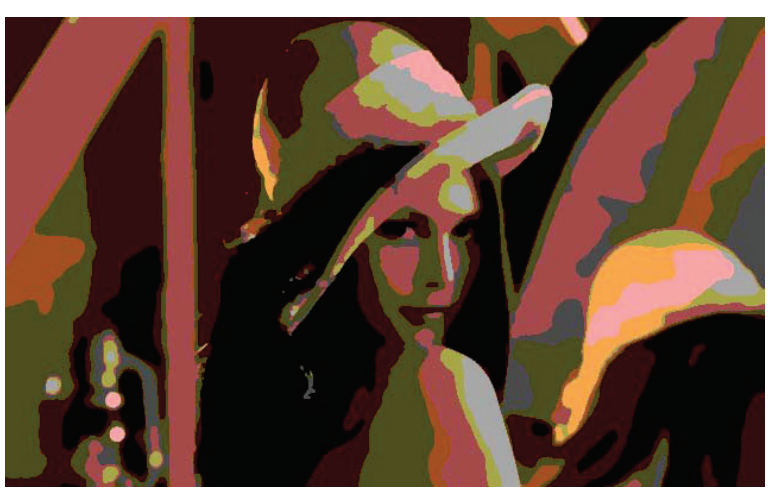

VML

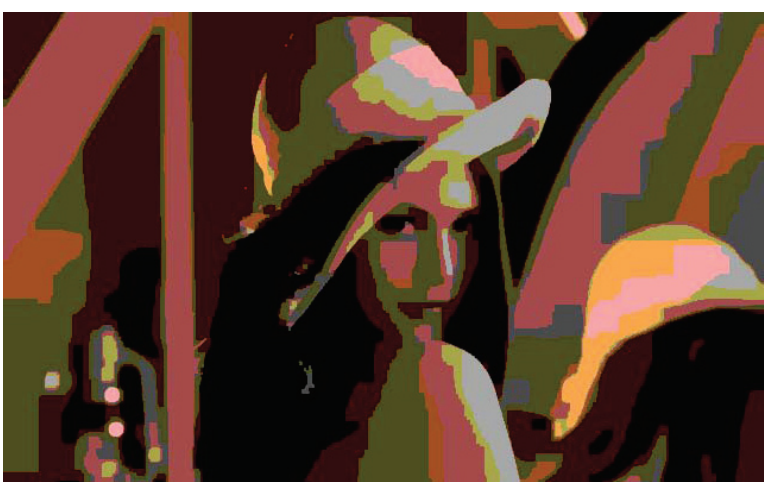

TRW-S

Figure 7. Closeups of $4 \times 4 \times 4$ RGB label segmentations (top) using different algorithms reveal a typical problem of discrete approaches: they exhibit a preference for horizontal and vertical region boundaries since they penalize an $L^{1}$-distance instead of the correct Euclidean length [18, 42]. The error can be reduced by increasing the neighborhood connectivity, but only at extremely high costs of memory and computation time. Overall, these metrication errors lead to blocky and visually less pleasing segmentation results (bottom).

From a theoretical point of view, the move-making schemes $\alpha$-EXP and SWAP solve the problem by iterating binary decisions instead of dealing with the whole problem at once, which explains their efficiency. However, as a result they cannot give reasonable optimality bounds; see remarks in Table 2. As an additional drawback, all discrete methods suffer from metrication errors [18, 42], as detailed in Figure 7.

7.2. Adaptive denoising. As a novel application of a multidimensional label space, we present adaptive denoising, where we jointly estimate a noise level and a denoised image by solving a single minimization problem. Note that here we require the continuous label space to represent the image intensity range.

The Mumford-Shah energy can be interpreted as a denoising model which yields the maximum a posteriori estimate for the original image under the assumption that the input image $f$ was distorted with Gaussian noise of standard deviation $\sigma$. An interesting generalization of this model is when the standard deviation of the noise is not constant but rather varies over the image. Viewing it as an additional unknown, the label space becomes two-dimensional, with one dimension representing the unknown intensity $u$ of the original image, and the second dimension representing the unknown standard deviation $\sigma$ of the noise. The data term of the energy can then be written as [7]

$$
\int_{\Omega} \frac{(u-f)^{2}}{2 \sigma^{2}}+\frac{1}{2} \log \left(2 \pi \sigma^{2}\right) \mathrm{d} x .
$$




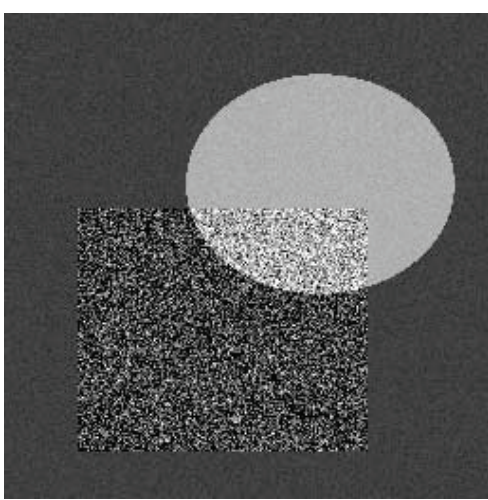

Input, spatially varying noise
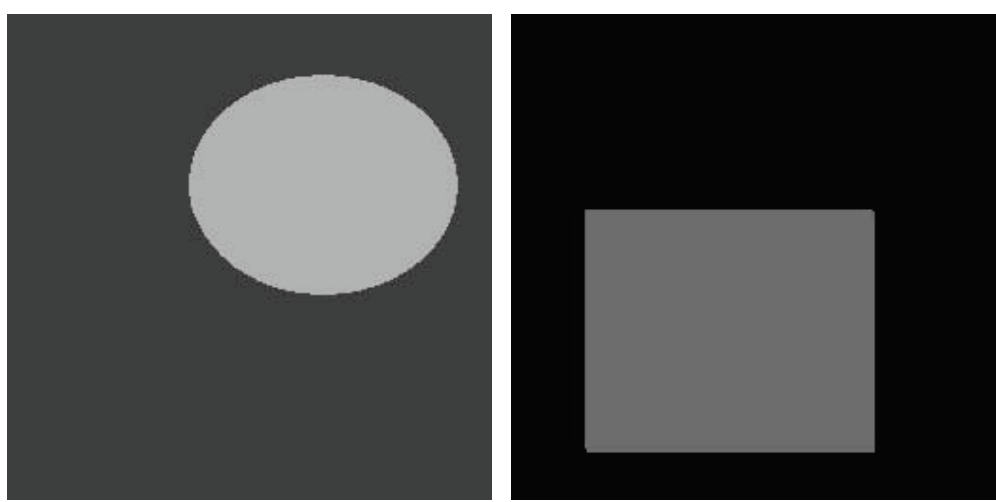

Denoised image and reconstructed standard deviation

Figure 8. The algorithm allows us to jointly recover the unknown standard deviation $\sigma$ of the noise as well as the intensity of a denoised image by solving a single optimization problem. Ground truth: within rectangle Gaussian noise with standard deviation $\sigma=0.25$, outside $\sigma=0.02$; image intensity within ellipsoid $u=0.7$, outside $u=0.3$. Image resolution is $256 \times 256$ using $32 \times 32$ labels. Computation time is 4.4 minutes. Reprinted with permission from [36, Figure 4], (C) 2011 by IEEE Computer Society, Washington, DC.

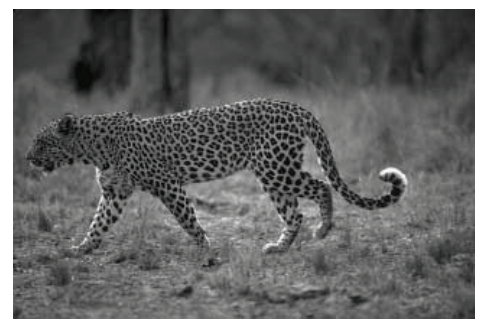

Input, textured object

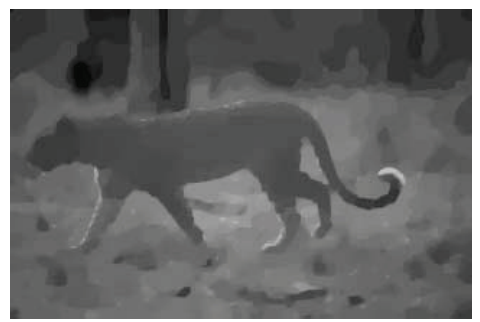

Simultaneous piecewise smooth approximation of intensity (left) and standard deviation (right)

Figure 9. A piecewise smooth image approximation of both intensity and noise standard deviation using (7.2) and the Mumford-Shah regularizer for both $u$ and $\sigma$. This model allows us to separate textured objects in a natural way by jointly estimating the mean and standard deviation of image intensities. The amount of smoothing is stronger in the region of larger standard deviation. Image resolution is $320 \times 214$ using $32 \times 32$ labels, leading to a run time of 10.3 minutes.

Results of the optimization can be observed in Figures 8 and 9. For the regularizer, we used piecewise constant Mumford-Shah for both $\sigma$ and $u$ in Figure 8, and piecewise smooth Mumford-Shah in Figure 9. In the real-world example Figure 9, the solution can be interpreted as a uniformly smooth approximation, where all regions attain a similar smoothness level regardless of the amount of texture in the input.

7.3. Depth and occlusion map. In this test, we simultaneously compute a depth map and an occlusion map for a stereo pair of two color input images $I_{L}, I_{R}: \Omega \rightarrow \mathbb{R}^{3}$. The occlusion map shall be a binary map denoting whether a pixel in the left image has a matching pixel in the right image. Thus, the space of labels is two-dimensional with $\Lambda_{1}$ consisting of the disparity values and a binary $\Lambda_{2}=\{0,1\}$ for the occlusion map. We use the TV smoothness penalty on the disparity values. The Potts regularizer is imposed for the occlusion map. The 

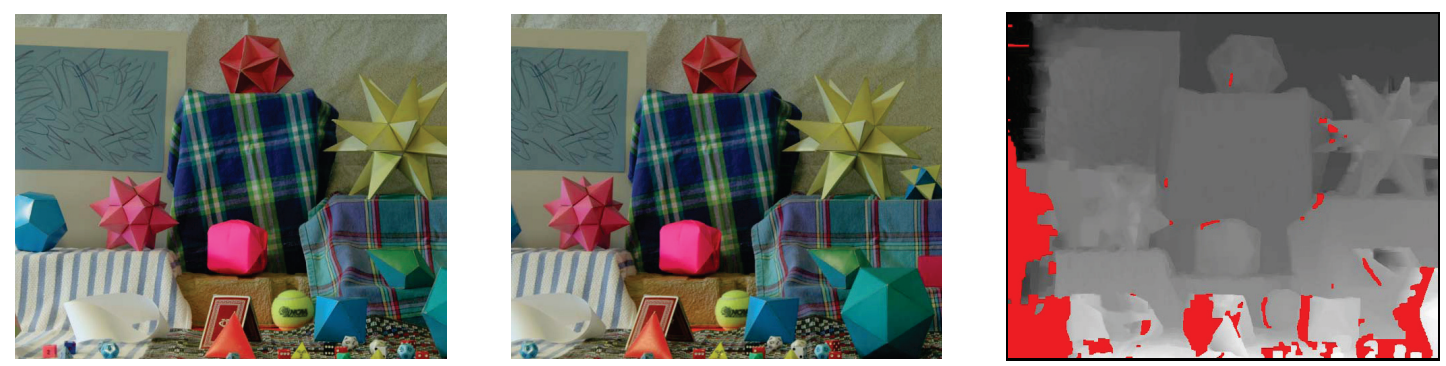

Figure 10. The proposed method can be employed to simultaneously optimize for a displacement and an occlusion map. This problem is also too large to be solved by alternative relaxation methods on current GPUs. From left to right: left and right input images $I_{L}$ and $I_{R}$, and computed disparity and occlusion map; red areas denote occluded pixels. Reprinted with permission from [15, Figure 6], (C) 2010 by Springer-Verlag, Berlin, Heidelberg.

distance on the label space thus becomes

$$
d(\gamma, \chi)=s_{1}\left|\gamma_{1}-\chi_{1}\right|+s_{2}\left|\gamma_{2}-\chi_{2}\right|
$$

with suitable weights $s_{1}, s_{2}>0$. We penalize an occluded pixel with a constant $\operatorname{cost} c_{o c c}>0$, which corresponds to a threshold for the similarity measure above which we believe that a pixel is no longer matched correctly. The cost associated with a label $\gamma$ at $(x, y) \in \Omega$ is then defined as

$$
c^{\gamma}(x, y)= \begin{cases}c_{o c c} & \text { if } \gamma_{2}=1, \\ \left|I_{L}(x, y)-I_{R}\left(x-\gamma_{1}, y\right)\right|_{2} & \text { otherwise. }\end{cases}
$$

The result for the "Moebius" test pair from the Middlebury benchmark is shown in Figure 10. The input image resolution was scaled to $640 \times 512$, requiring 128 disparity labels, which resulted in a total memory consumption which was slightly too big for previous methods but still in reach of the proposed algorithm. Total computation time required was 1170 seconds.

7.4. Optic flow. In this experiment, we compute optic flow between two color input images $I_{0}, I_{1}: \Omega \rightarrow \mathbb{R}^{3}$ taken at two different time instants. The space of labels is again two-dimensional, with $\Lambda_{1}=\Lambda_{2}$ denoting the possible components of flow vectors in the $x$ and $y$-direction, respectively. We regularize both directions with either TV or a truncated linear penalty on the component distance, i.e.,

$$
d(\gamma, \chi)=s \min \left(t,\left|\gamma_{1}-\chi_{1}\right|\right)+s \min \left(t,\left|\gamma_{2}-\chi_{2}\right|\right),
$$

with a suitable manually chosen weight $s>0$ and threshold $t>0$. Note that we can provide a tight relaxation of the exact penalizer, which was only coarsely approximated in the previous approaches $[15,22]$. The cost function just compares pointwise pixel colors in the images; i.e.,

$$
c^{\gamma}(x, y)=\left|I_{0}(x, y)-I_{1}\left(x+\gamma_{1}, y+\gamma_{2}\right)\right|_{2} .
$$

Results can be observed in Figures 1, 11, 12, and 13. See Figure 12 for the color code of the flow vectors. In all examples, the number of labels is so high that this problem is currently impossible to solve with previous convex relaxation techniques by a large margin; see Table 1. 


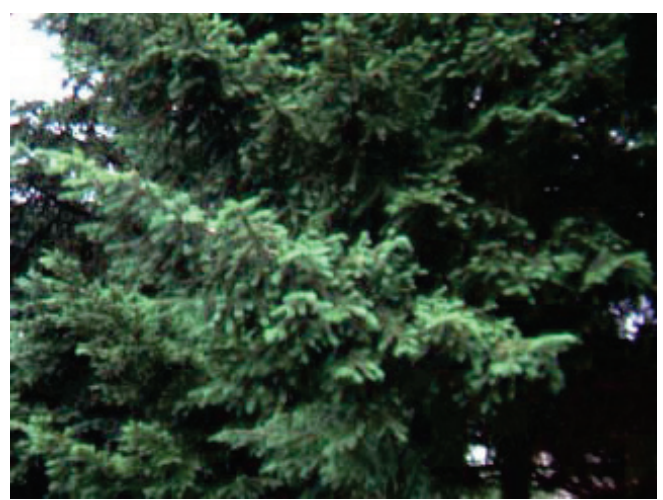

First image $I_{0}$

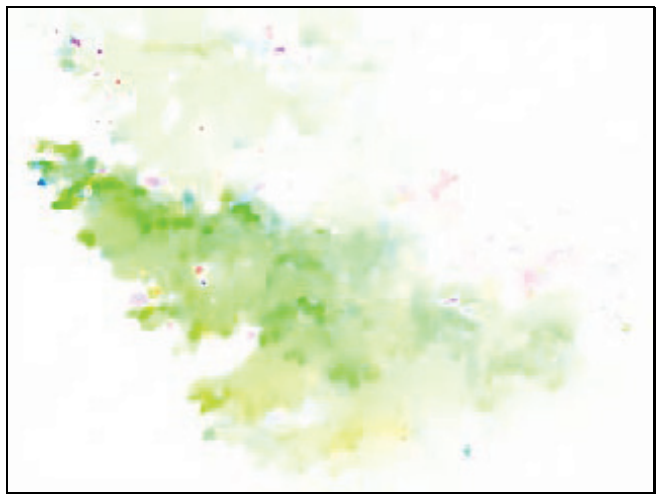

Previous relaxation

(25 minutes run time)

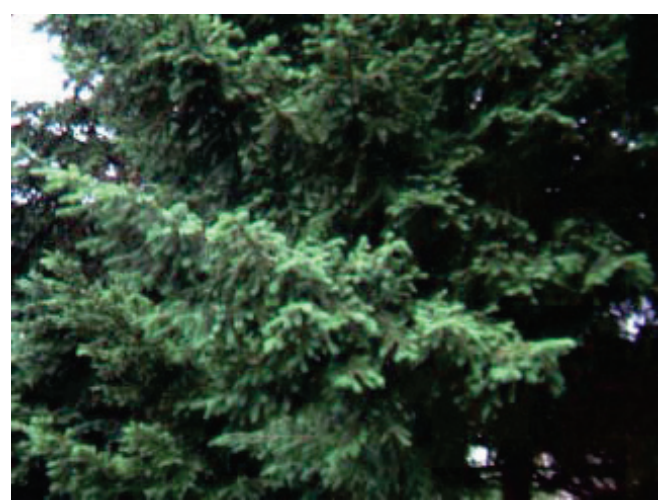

Second image $I_{1}$

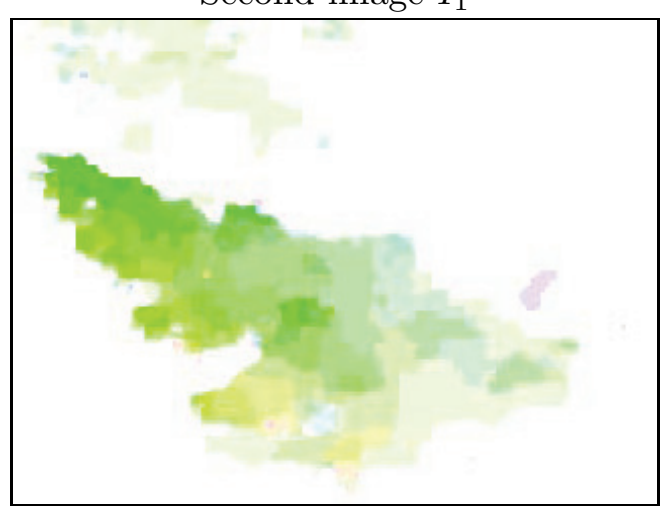

Proposed relaxation

(2 minutes run time)

Figure 11. Optical flow fields with $32 \times 32$ labels computed on an image with resolution $320 \times 240$ using TV regularization. With the new relaxation of the regularizers, we achieve optimality bounds which are on average three times lower than with previous relaxations from [15, 22], using the proposed data term relaxation (4.10) for both cases. The result in the lower left is computed with the TV relaxation from [22]. Since the scaling of the regularity term is not directly comparable, we chose optimal parameters for both algorithms manually. The large time difference results from a narrow constraint on the time step; see section 7.4. Reprinted with permission from [36, Figure 3], (C) 2011 by IEEE Computer Society, Washington, DC.

Compared to the relaxation proposed in the original conference publication [15], total computation time was reduced dramatically; see Figure 11. The large computation time for the TV relaxation of [15] is caused by an overly restrictive constraint on the time steps due to the structure of the embedding matrix $A_{k}$ in (5.3). Using $N_{1}=N_{2}:=N$ labels in each direction of the two-dimensional optic flow label space, the time steps can be seen to be proportional to $N^{-3 / 2}$. In contrast, the proposed TV relaxation in section 5.3 allows larger time steps proportional to $N^{-1 / 2}$ and thus leads to a substantially lower number of iterations. We suggest using the preconditioning variant of the algorithm [10] where the time steps are chosen adaptively.

Due to the global optimization of a convex energy, we can successfully capture large displacements without having to implement a coarse-to-fine scheme, see Figure 12. Table 3 shows detailed numeric results of our method on the data sets of the Middlebury benchmark 


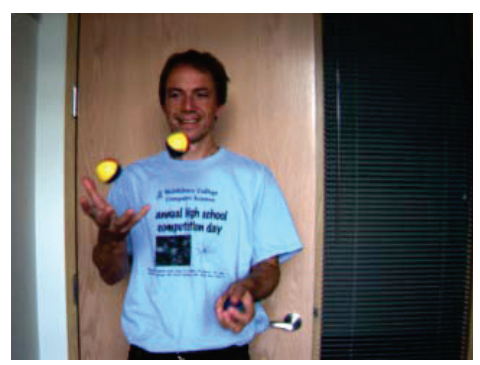

First image $I_{0}$

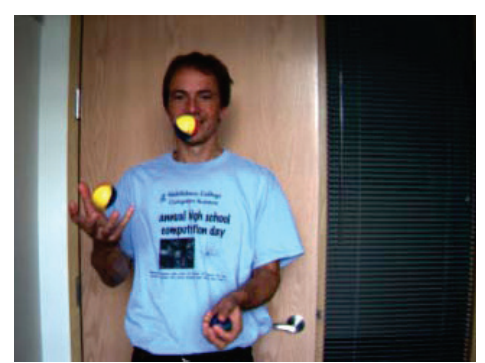

Second image $I_{1}$

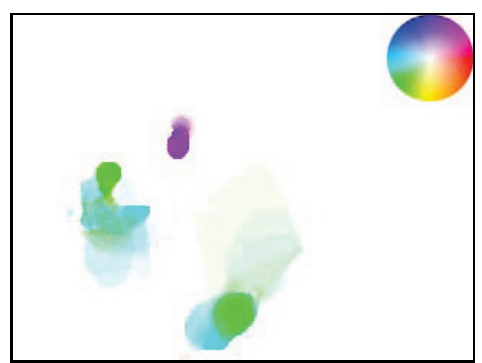

Flow field and color code

Figure 12. When employed for optic flow, the proposed method can successfully capture large displacements without the need for coarse-to-fine approaches, since a global optimization is performed over all labels. In contrast to existing methods, our solution is within a known bound of the global optimum. Reprinted with permission from [15, Figure 7], (c) 2010 by Springer-Verlag, Berlin, Heidelberg.

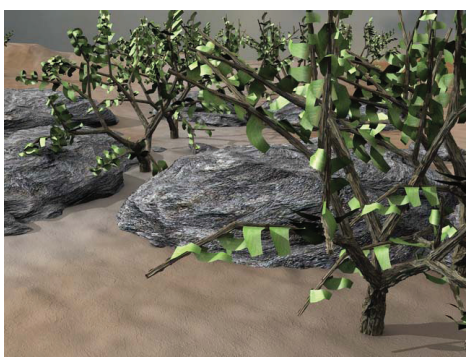

First image $I_{0}$

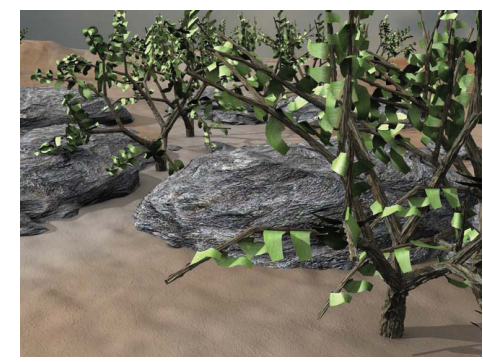

Second image $I_{1}$

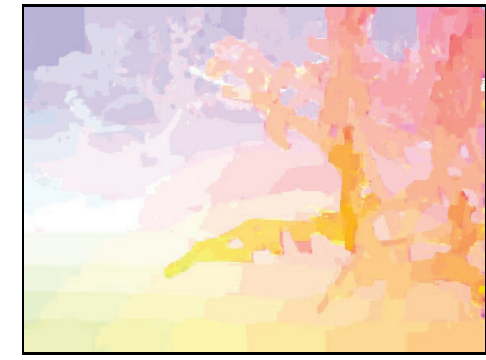

Flow field

Figure 13. Example with a larger image resolution of $640 \times 480$ pixels, which requires $32 \times 32$ labels. The regularizer is the $T V$ in each component. Computation time is 21.6 minutes. Reprinted with permission from [36, Figure 1], (c) 2011 by IEEE Computer Society, Washington, DC.

with public ground truth available. We compare our current method with a linear regularizer using $35 \times 35$ labels to our old relaxation [15] and TV- $L^{1}$ optic flow [43], which utilizes a very similar energy which is optimized with a coarse-to-fine scheme and quadratic relaxation of the linearized functional. Results show that we get reasonable optimality bounds for the energy and are in most cases within $5 \%$ of the global optimum, while accuracy of the actual optical flow results depends on how fine the discretization is compared to the maximum displacement. The new method is obviously superior to the old relaxation in all respects - the previous one is provided only for reference, and we strongly recommend using the new one proposed in this work. For a method which is competitive on the Middlebury benchmark, we would need to further increase the number of labels, by, e.g., implementing a coarse-to-fine scheme, and fine-tune our data terms. This is, however, beyond the scope of this paper, since our focus is to provide an efficient optimization framework.

8. Conclusion. We have introduced a continuous convex relaxation for multilabel problems where the label space has a product structure and the regularizer is separable. Such labeling problems are plentiful in computer vision. The proposed reduction method improves on previous methods in that it requires orders of magnitude less memory and computation 
Table 3

Accuracy comparison on Middlebury data sets. Maximum displacement $(\Delta)$ and average endpoint error (aep) are measured in pixels, and average angular error (aan) in degrees. Not surprisingly, accuracy for the VML method is strongly correlated with the number of labels per pixel and thus decreases with larger maximum displacement. On data sets with small maximum displacement, the accuracy using $35 \times 35$ labels is comparable to that of $T V-L^{1}$ optical flow, while other data sets would require either coarse-to-fine schemes or a greater number of labels for the method to remain competitive. The proposed new relaxation outperforms the previous one [15] in all respects.

\begin{tabular}{|c|c|c|c|c|c|c|c|c|c|}
\hline \multicolumn{2}{|l|}{ Data set } & \multicolumn{3}{|c|}{ VML } & \multicolumn{3}{|c|}{ VML-ECCV [15] } & \multicolumn{2}{|c|}{ TV- $L^{1}[43]$} \\
\hline size & $\Delta$ & aep & aan & bound [\%] & aep & aan & bound [\%] & aep & aan \\
\hline $\begin{array}{c}\text { Venus } \\
420 \times 380\end{array}$ & 10 & 0.39 & 4.25 & 0.21 & 0.81 & 5.44 & 5.88 & 0.44 & 7.74 \\
\hline $\begin{array}{l}584 \times 388 \\
\text { Hydrangea }\end{array}$ & 5 & 0.22 & 4.58 & 6.12 & 0.62 & 6.38 & 7.87 & 0.22 & 3.94 \\
\hline $\begin{array}{l}584 \times 388 \\
\text { RubberWha }\end{array}$ & $\begin{array}{l}12 \\
\text { ale }\end{array}$ & 0.42 & 4.06 & 2.64 & 0.81 & 5.60 & 9.26 & 0.22 & 2.64 \\
\hline $\begin{aligned} & 584 \times 388 \\
& \text { Grove } 2\end{aligned}$ & 5 & 0.18 & 5.73 & 2.36 & 0.29 & 6.12 & 8.60 & 0.20 & 6.29 \\
\hline $\begin{array}{l}640 \times 480 \\
\quad \text { Grove3 }\end{array}$ & 5 & 0.34 & 4.54 & 5.01 & 0.55 & 6.16 & 13.25 & 0.22 & 3.12 \\
\hline $\begin{array}{c}640 \times 480 \\
\text { Urban2 }\end{array}$ & 15 & 1.06 & 12.02 & 9.22 & 2.01 & 14.49 & 10.50 & 0.76 & 7.41 \\
\hline $\begin{array}{c}640 \times 480 \\
\text { Urban3 }\end{array}$ & 22 & 0.81 & 9.31 & 1.07 & 0.97 & 8.15 & 6.32 & 0.47 & 3.51 \\
\hline $640 \times 480$ & 18 & 1.38 & 8.95 & 1.05 & 1.65 & 10.82 & 4.99 & 0.90 & 8.02 \\
\hline
\end{tabular}

time, while retaining the advantages: a very flexible choice of regularizer on the label space, a globally optimal solution of the relaxed problem, and an efficient parallel GPU implementation with guaranteed convergence.

The proposed framework combines the advantages of the efficient multidimensional data term relaxation [36] with the tight relaxation of the regularizers in [9]. It allows for a very general class of continuous regularizers on multidimensional label spaces and can thus efficiently solve a significant range of problems. For example, we can explicitly encourage the solution to be smooth in certain regions and can represent Huber-TV and truncated linear regularization by an exact and tight relaxation. The regularizers can be arbitrarily mixed, in the sense that each dimension of the label space can have its own type of regularity. Because of the reduced memory requirements, we can successfully handle specific problems with very large numbers of labels, which could not be done with previous labeling methods. A systematic experimental comparison with discrete algorithms ( $\alpha$-EXP, SWAP, BP, TRW-S) shows a good performance and often improved results.

\section{Appendix: Proofs of propositions and theorems in the main paper.}

\subsection{Proof of Proposition 3.1.}

Proof. In order to prove the proposition, we show that the mapping induces a pointwise bijection from $\Delta_{\times}$onto $\Delta$. We first show that it is onto: for $\boldsymbol{u}(x)$ in $\Delta$, there exists exactly one $\gamma \in \Gamma$ with $u^{\gamma}(x)=1$. Set $v_{k}^{\lambda}(x)=1$ if $\lambda=\gamma_{k}$, and $v_{k}^{\lambda}(x)=0$ otherwise. Then (3.5) is satisfied as desired; see Figure 2. To see that the map is one-to-one, we just count the elements in $\Delta_{\times}$. Since $\Delta_{k}$ contains $N_{k}$ elements, the number of elements in $\Delta_{\times}$is $N_{1} \times \cdots \times N_{d}=N$, the same as in $\Delta$. 


\subsection{Proof of Theorem 4.1.}

Proof. Since derivatives of indicator functions do not exist in an ordinary sense, the integral in (4.1) is meant to be a convenient notation for

$$
\int_{\Omega \times \Lambda_{k}}\left(\phi^{1}, \phi^{2}\right) \cdot \nu_{\Gamma_{u_{k}}} \mathrm{~d} \mathcal{H}^{n}(x, \lambda)
$$

where

$$
\Gamma_{u_{k}}:=\left\{(x, u(x)) \mid x \in \Omega \backslash S_{u_{k}}\right\} \cup\left\{(x, s) \mid x \in S_{u_{k}}, s \in\left[u_{k}^{-}, u_{k}^{+}\right]\right\}
$$

is the extended graph of $u$, and $\nu_{\Gamma_{u_{k}}}$ is the normal on $\Gamma_{u_{k}}$ pointing "downwards." Intuitively, $\nabla 1_{\text {hyp }\left(u_{k}\right)}$ in (4.1) is nonzero only on $\Gamma_{u_{k}}$ and equals $\nu_{\Gamma_{u_{k}}}$ up to a delta function factor. For a fixed $\phi$ denote the integral (9.1) by $J_{\phi}$. It is equal to [2, Lemma 2.8]

$$
\begin{aligned}
J_{\phi}=\int_{\Omega \backslash S_{u_{k}}}( & \left.\phi^{1}\left(x, u_{k}\right) \cdot \nabla u_{k}-\phi^{2}\left(x, u_{k}\right)\right) \mathrm{d} x \\
& \quad+\int_{S_{u_{k}}}\left(\int_{u_{k}^{-}}^{u_{k}^{+}} \phi^{1}(x, s) \mathrm{d} s\right) \cdot \nu_{u_{k}} \mathrm{~d} \mathcal{H}^{n-1}(x) .
\end{aligned}
$$

Define $\boldsymbol{p}: \Omega \times \Lambda_{k} \rightarrow \mathbb{R}^{n}$ and $b: \Omega \times \Lambda_{k} \rightarrow \mathbb{R}$ by

$$
\boldsymbol{p}(x, \lambda):=\int_{\lambda_{0}}^{\lambda} \phi^{1}(x, s) \mathrm{d} s, \quad b(x, \lambda):=\phi^{2}(x, \lambda)
$$

for some $\lambda_{0} \in \Lambda_{k}$. Since $\phi \in C_{c}^{1}\left(\Omega \times \Lambda_{k} ; \mathbb{R}^{n} \times \mathbb{R}\right)$, also $(p, b) \in C_{c}^{1}\left(\Omega \times \Lambda_{k} ; \mathbb{R}^{n} \times \mathbb{R}\right)$. With these new variables we have

$$
\begin{aligned}
J_{\phi}=\int_{\Omega \backslash S_{u_{k}}}\left(\partial_{\lambda} \boldsymbol{p}\left(x, u_{k}\right) \cdot \nabla u_{k}-b\left(x, u_{k}\right)\right) \mathrm{d} x \\
\quad+\int_{S_{u_{k}}}\left(\boldsymbol{p}\left(x, u_{k}^{+}\right)-\boldsymbol{p}\left(x, u_{k}^{-}\right)\right) \cdot \nu_{u_{k}} \mathrm{~d} \mathcal{H}^{n-1}(x) .
\end{aligned}
$$

By the divergence theorem,

$$
\begin{gathered}
\int_{\Omega \backslash S_{u_{k}}} \operatorname{div}\left(\boldsymbol{p}\left(x, u_{k}\right)\right) \mathrm{d} x=\int_{S_{u_{k}}}\left(\boldsymbol{p}\left(x, u_{k}^{+}\right) \cdot\left(-\nu_{u_{k}}\right)+\boldsymbol{p}\left(x, u_{k}^{-}\right) \cdot \nu_{u_{k}}\right) \mathrm{d} \mathcal{H}^{n-1}(x) \\
+\int_{\partial \Omega} \boldsymbol{p}\left(x, u_{k}\right) \cdot \nu_{\partial \Omega} \mathrm{d} \mathcal{H}^{n-1}(x) .
\end{gathered}
$$

In the integrand of the first integral on the right-hand side there are two addends for each point of $S_{u_{k}}$, because the integration on the left-hand side is performed on both sides of $S_{u_{k}}$. The outer normal for the $u_{k}^{-}$side is $\nu_{u_{k}}$ by definition, and for the $u_{k}^{+}$side it is just the opposite. The last integral on the right-hand side is zero because $\phi$ and therefore also $\boldsymbol{p}$ have compact support in $\Omega$. Using (9.6) in (9.5) we obtain

$$
J_{\phi}=\int_{\Omega \backslash S_{u_{k}}}\left(\partial_{\lambda} \boldsymbol{p}\left(x, u_{k}\right) \cdot \nabla u_{k}-b\left(x, u_{k}\right)\right) \mathrm{d} x-\int_{\Omega \backslash S_{u_{k}}} \operatorname{div}\left(\boldsymbol{p}\left(x, u_{k}\right)\right) \mathrm{d} x .
$$


By the chain rule,

$$
\operatorname{div}\left(\boldsymbol{p}\left(x, u_{k}\right)\right)=(\operatorname{div} \boldsymbol{p})\left(x, u_{k}\right)+\partial_{\lambda} \boldsymbol{p}\left(x, u_{k}\right) \cdot \nabla u_{k} .
$$

Thus, the expression (9.7) simplifies to

$$
J_{\phi}=\int_{\Omega \backslash S_{u_{k}}}\left(-(\operatorname{div} \boldsymbol{p})\left(x, u_{k}\right)-b\left(x, u_{k}\right)\right) \mathrm{d} x=\int_{\Omega \times \Lambda_{k}}(-\operatorname{div} \boldsymbol{p}-b) \boldsymbol{v}_{k} \mathrm{~d}(x, \lambda) .
$$

The last equality is simply the definition of how the distribution $\boldsymbol{v}_{k}(x, \lambda)=\delta\left(u_{k}-\lambda\right)$, defined for $u_{k} \in \mathcal{S B V}(\Omega)$, acts on functions. Now, the claim of the proposition follows directly from (4.1) and (9.9).

\subsection{Proof of Proposition 4.2.}

Proof. Since at each point $x \in \Omega, \gamma(x)$ is the label indicated by $\boldsymbol{v}(x)$, by the defining property (3.8) we have $v_{k}^{\lambda}(x)=1$ for $\lambda=\gamma_{k}(x)$ and $v_{k}^{\lambda}(x)=0$ for all $\lambda \in \Lambda_{k}$ with $\lambda \neq \gamma_{k}(x)$. Thus, for all $\boldsymbol{q} \in \mathcal{Q}$,

$$
\sum_{k=1}^{d} \sum_{\lambda \in \Lambda_{k}} q_{k}^{\lambda}(x) v_{k}^{\lambda}(x)=\sum_{k=1}^{d} q_{k}^{\gamma_{k}(x)} \leq c^{\gamma(x)}(x)
$$

This shows that at least $R_{\text {data }}(\boldsymbol{v}) \leq \int_{\Omega} c^{\gamma(x)}(x) \mathrm{d} x=E_{\text {data }}(\boldsymbol{v})$. To prove equality, first observe that we can safely interchange the supremum over $\boldsymbol{q} \in \mathcal{Q}$ with the integration over $\Omega$ since the constraints in $\mathcal{Q}$ on $\boldsymbol{q}$ are pointwise in $x$. This means that we need only show the pointwise equality, i.e., that for each fixed $x \in \Omega$ the integrand in (4.10) yields $c^{\gamma}(x)$ when taking the supremum over $\boldsymbol{q} \in \mathcal{Q}$. We use Lagrange multipliers $\boldsymbol{\mu}$ to write the constraints in (4.11) as additional energy terms, interchanging the ordering of $\sup _{\boldsymbol{q}}$ and $\inf _{\boldsymbol{\mu}}$ :

$$
\begin{aligned}
R_{\text {data }}(\boldsymbol{v}) & =\sup _{\boldsymbol{q} \in \mathcal{Q}} \sum_{k=1}^{d} \sum_{\lambda \in \Lambda_{k}} q_{k}^{\lambda} v_{k}^{\lambda} \\
& =\sup _{\boldsymbol{q}} \inf _{\mu^{\widehat{\gamma}} \geq 0} \sum_{k=1}^{d} \sum_{\lambda \in \Lambda_{k}} q_{k}^{\lambda} v_{k}^{\lambda}-\sum_{\widehat{\gamma} \in \Gamma} \mu^{\widehat{\gamma}}\left(q_{1}^{\widehat{\gamma}}+\cdots+q_{d}^{\widehat{\gamma}_{d}}-c^{\widehat{\gamma}}\right) \\
& =\inf _{\mu^{\gamma} \geq 0} \sum_{\widehat{\gamma} \in \Gamma} \mu^{\widehat{\gamma}} c^{\widehat{\gamma}}+\sup _{\boldsymbol{q}} \sum_{k=1}^{d} \sum_{\lambda \in \Lambda_{k}} q_{k}^{\lambda}\left(v_{k}^{\lambda}-\sum_{\widehat{\gamma} \in \Gamma: \widehat{\gamma}_{k}=\lambda} \mu^{\widehat{\gamma}}\right) .
\end{aligned}
$$

Evaluating the supremum over $\boldsymbol{q}$ leads to constraints on the variables $\mu^{\widehat{\gamma}}$, and we obtain

$$
R_{\text {data }}(\boldsymbol{v})=\inf _{\mu^{\widehat{\gamma}} \geq 0} \sum_{\widehat{\gamma} \in \Gamma} \mu^{\widehat{\gamma}} c^{\widehat{\gamma}}
$$

with $\mu^{\widehat{\gamma}}$ such that additionally

$$
\sum_{\widehat{\gamma} \in \Gamma: \widehat{\gamma}_{k}=\lambda} \mu^{\widehat{\gamma}}=v_{k}^{\lambda} \quad \text { for all } 1 \leq k \leq d \text { and } \lambda \in \Lambda_{k}
$$


First, for any fixed $1 \leq k \leq d$ and any $\lambda \in \Lambda_{k}$ with $\lambda \neq \gamma_{k}$, by assumption we have $v_{k}^{\lambda}=0$. Since $\mu^{\widehat{\gamma}} \geq 0$, (9.13) then gives $\mu^{\widehat{\gamma}}=0$ for all $\widehat{\gamma} \in \Gamma$ with $\widehat{\gamma}_{k} \neq \gamma_{k}$. Combining this for all $1 \leq k \leq d$, we get $\mu^{\widehat{\gamma}}=0$ for all $\widehat{\gamma} \neq \gamma$. Next, plug $\lambda=\gamma_{k}$ for some $k$ into (9.13). Since any other addend $\mu^{\widehat{\gamma}}$ is zero, the sum is just $\mu^{\gamma}$, while the right-hand side is $v_{k}^{\gamma_{k}}=1$.

Therefore, the constraints (9.13) ensure that $\mu^{\widehat{\gamma}}=0$ for all $\widehat{\gamma} \neq \gamma$ and $\mu^{\gamma}=1$, so that (9.12) gives $R_{\text {data }}(\boldsymbol{v})=c^{\gamma}$.

\subsection{Proof of Theorem 4.3.}

Proof. Let $\boldsymbol{v} \in \mathcal{L}^{2}\left(\Omega, \operatorname{co}\left(\Delta_{\times}\right)\right)$be arbitrary, and set $q_{k}^{\lambda}(x):=\hat{c}(x) / d$. Then

$$
\sum_{k=1}^{d} \sum_{\lambda \in \Lambda_{k}} q_{k}^{\lambda}(x) v_{k}^{\lambda}(x)=\sum_{k=1}^{d} \frac{\hat{c}(x)}{d} \sum_{\lambda \in \Lambda_{k}} v_{k}^{\lambda}(x)=\sum_{k=1}^{d} \frac{\hat{c}(x)}{d}=\hat{c}(x),
$$

and $\sum_{k} q_{k}^{\gamma_{k}}(x)=\hat{c}(x) \leq c^{\gamma}(x)$ for all $\gamma \in \Gamma$ and $x \in \Omega$, so $\boldsymbol{q} \in \mathcal{Q}$. This shows that $R_{\text {data }}(\boldsymbol{v}) \geq$ $\int_{\Omega} \hat{c}(x) \mathrm{d} x$, which is the minimum of $E_{\text {data }}$ for binary functions.

\subsection{Proof of Proposition 4.4.}

Proof. By convex duality [30], the convex envelope of $E_{\text {data }}$ is given by the LegendreFenchel biconjugate $E_{\text {data }}^{* *}$. The first convex conjugate $E_{\text {data }}^{*}$ of $E_{\text {data }}$ is given as

$$
E_{\text {data }}^{*}(\boldsymbol{q})=\sup _{\boldsymbol{v}} \int_{\Omega} \sum_{k=1}^{d} \sum_{\lambda \in \Lambda_{k}} q_{k}^{\lambda}(x) v_{k}^{\lambda}(x) \mathrm{d} x-E_{\text {data }}(v) .
$$

Since $E_{\text {data }}$ is finite only for binary $\boldsymbol{v}$, i.e., if $v_{k}^{\lambda}(x)=\chi_{\lambda=\gamma_{k}(x)}$ for some $\gamma: \Omega \rightarrow \Gamma$, this reduces to

$$
F(\boldsymbol{q}):=E_{\text {data }}^{*}(\boldsymbol{q})=\sup _{\gamma: \Omega \rightarrow \Gamma} \int_{\Omega}\left(\sum_{k=1}^{d} q_{k}^{\gamma_{k}(x)}(x)-c^{\gamma(x)}(x)\right) \mathrm{d} x .
$$

The biconjugate is then

$$
E_{\text {data }}^{* *}(\boldsymbol{v})=\sup _{\boldsymbol{q}} \int_{\Omega} \sum_{k=1}^{d} \sum_{\lambda \in \Lambda_{k}} q_{k}^{\lambda}(x) v_{k}^{\lambda}(x) \mathrm{d} x-F(q) .
$$

For $\boldsymbol{q} \in \mathcal{L}^{2}\left(\Omega, \mathbb{R}^{N_{1}+\cdots+N_{d}}\right)$ and $a \in \mathcal{L}^{2}(\Omega, \mathcal{R})$, define $\boldsymbol{q}_{a} \in \mathcal{L}^{2}\left(\Omega, \mathbb{R}^{N_{1}+\cdots+N_{d}}\right)$ by $\left(q_{a}\right)_{k}^{\lambda}(x):=$ $q_{k}^{\lambda}(x)+a_{k}(x)$. Then obviously

$$
F\left(\boldsymbol{q}_{a}\right)=F(\boldsymbol{q})+\int_{\Omega} \sum_{k=1}^{d} a_{k}(x) d x .
$$

Inserting $\boldsymbol{q}_{a}$ for $\boldsymbol{q}$ in (9.17), we obtain

$$
E_{\text {data }}^{* *}(\boldsymbol{v})=\sup _{\boldsymbol{q}, a} \int_{\Omega} \sum_{k=1}^{d} \sum_{\lambda \in \Lambda_{k}} q_{k}^{\lambda}(x) v_{k}^{\lambda}(x) \mathrm{d} x-F(q)+\int_{\Omega} \sum_{k=1}^{d} a_{k}(x)\left(\sum_{\lambda \in \Lambda_{k}} v_{k}^{\lambda}(x)-1\right) \mathrm{d} x .
$$


Holding $\boldsymbol{q}$ fixed and taking the supremum over $a$, we see that in order for $E_{\text {data }}^{* *}(v)$ to be finite, we necessarily must have

$$
\sum_{\lambda \in \Lambda_{k}} v_{k}^{\lambda}(x)=1 \quad \text { for all } 1 \leq k \leq d, \text { for a.e. } x \in \Omega .
$$

We assume these equalities from now on, i.e., that $\boldsymbol{v} \in \mathcal{S}$ with $\mathcal{S}$ as defined in the proposition.

In the same way as we arrived at (9.19), we see that given (9.20) the expression in (9.17), over which the supremum is taken, does not change if we replace $\boldsymbol{q}$ by $\boldsymbol{q}_{a}$ for some $a$. Also, (9.18) shows that for each $\boldsymbol{q}$ and any fixed $\alpha \in \mathbb{R}$ we can find an $a$ with $F\left(\boldsymbol{q}_{a}\right)=\alpha$. Combining these two observations, we obtain

$$
E_{\text {data }}^{* *}(\boldsymbol{v})=\sup _{\boldsymbol{q}: F(\boldsymbol{q})=\alpha} \int_{\Omega} \sum_{k=1}^{d} \sum_{\lambda \in \Lambda_{k}} q_{k}^{\lambda}(x) v_{k}^{\lambda}(x) \mathrm{d} x-\alpha .
$$

Adding $\alpha$ on both sides of the equation and taking the supremum over all $\alpha \leq 0$, we get

$$
E_{\text {data }}^{* *}(\boldsymbol{v})=\sup _{\boldsymbol{q}: F(\boldsymbol{q}) \leq 0} \int_{\Omega} \sum_{k=1}^{d} \sum_{\lambda \in \Lambda_{k}} q_{k}^{\lambda}(x) v_{k}^{\lambda}(x) \mathrm{d} x .
$$

This is almost the expression (4.10) for $R_{\text {data }}(\boldsymbol{v})$. We need only replace the integral constraints on $\boldsymbol{q}$ in $F(\boldsymbol{q}) \leq 0$, with $F$ in (9.16), with pointwise constraints. For this, note that in (9.16) the supremum over $\gamma$ may be safely put inside the integral over $\Omega$ since the label space $\Gamma$ is finite (after the assumed discretization). Therefore, $F(\boldsymbol{q}) \leq 0$ is equivalent to

$$
\int_{\Omega} \sup _{\gamma \in \Gamma}\left(\sum_{k=1}^{d} q_{k}^{\gamma_{k}}(x)-c^{\gamma}(x)\right) \mathrm{d} x \leq 0 .
$$

Denoting the integrand by $a(x)$, this becomes equivalent to

$$
\exists a: \Omega \rightarrow \mathbb{R}: \quad \int_{\Omega} a(x) \mathrm{d} x \leq 0, \quad \sum_{k=1}^{d} q_{k}^{\gamma_{k}}(x)-c^{\gamma}(x) \leq a(x) \quad \text { for all } \gamma \in \Gamma, x \in \Omega .
$$

Given a $\boldsymbol{q}$ satisfying these constraints for some $a$, define $\widehat{\boldsymbol{q}}$ by $\widehat{q}_{k}^{\lambda}(x):=q_{k}^{\lambda}(x)-a(x) / d$. Then $\widehat{\boldsymbol{q}}$ satisfies (9.24) with $a \equiv 0$; i.e. $\widehat{\boldsymbol{q}} \in \mathcal{Q}$ with the constraint set $\mathcal{Q}$ in (4.11). Furthermore,

$$
\begin{aligned}
\int_{\Omega} \sum_{k=1}^{d} \sum_{\lambda \in \Lambda_{k}} \widehat{\boldsymbol{q}}_{k}^{\lambda}(x) v_{k}^{\lambda}(x) \mathrm{d} x & =\int_{\Omega} \sum_{k=1}^{d} \sum_{\lambda \in \Lambda_{k}}\left(q_{k}^{\lambda}(x)-\frac{a(x)}{d}\right) v_{k}^{\lambda}(x) \mathrm{d} x \\
& =\int_{\Omega} \sum_{k=1}^{d} \sum_{\lambda \in \Lambda_{k}} q_{k}^{\lambda}(x) v_{k}^{\lambda}(x) \mathrm{d} x-\int_{\Omega} a(x) \mathrm{d} x \\
& \geq \int_{\Omega} \sum_{k=1}^{d} \sum_{\lambda \in \Lambda_{k}} q_{k}^{\lambda}(x) v_{k}^{\lambda}(x) \mathrm{d} x
\end{aligned}
$$


using first (9.20) and then $\int_{\Omega} a(x) \leq 0$. Thus, among all $\boldsymbol{q}$ with $F(\boldsymbol{q}) \leq 0$ or, equivalently, with (9.24), the expression in the supremum (9.22) will be largest if we choose $a \equiv 0$ in (9.24). Hence,

$$
E_{\text {data }}^{* *}(\boldsymbol{v})=\sup _{\boldsymbol{q} \in \mathcal{Q}} \int_{\Omega} \sum_{k=1}^{d} \sum_{\lambda \in \Lambda_{k}} q_{k}^{\lambda}(x) v_{k}^{\lambda}(x) \mathrm{d} x=R_{\text {data }}(\boldsymbol{v})
$$

for $v \in \mathcal{S}$.

\subsection{Proof of Proposition 5.2.}

Proof. We can enforce a piecewise constant labeling $u_{k}$ if we enforce the approximate gradient $\nabla u_{k}$ to be constant zero. In (3.12), this can be achieved by setting $h_{k}\left(x, u_{k}(x), \nabla u_{k}(x)\right)=$ $c\left|\nabla u_{k}\right|$ with a constant $c>0$, and then letting $c \rightarrow \infty$ to enforce $\nabla u_{k} \equiv 0$ on $\Omega \backslash S_{u_{k}}$. Inserting the convex conjugate $h_{k}^{*}(x, \lambda, q)=\delta_{\{|q| \leq c\}}$, we find that the conditions in (4.4) now reduce to

$$
b^{\lambda} \geq 0, \quad\left|\partial_{\lambda} \boldsymbol{p}^{\lambda}\right|_{2} \leq c, \quad\left|\boldsymbol{p}^{\lambda}-\boldsymbol{p}^{\mu}\right|_{2} \leq d_{k}(\lambda, \mu)
$$

The supremum over $b^{\lambda} \geq 0$ is easily eliminated from (4.3) since $v_{k}^{\lambda} \geq 0$, i.e., $-b^{\lambda} v_{k}^{\lambda} \leq 0$ with 0 being the maximum possible value. The second constraint in (9.27) follows from the third if we choose $c \geq \max _{\lambda>\mu} \frac{d_{k}(\lambda, \mu)}{|\lambda-\mu|}$. Thus we arrive at (5.7) with the set $C_{k}$ as claimed in the proposition.

\subsection{Proof of Proposition 5.3.}

Proof. The claim follows from our general formulation (5.7) with a special choice of the dual variables $\boldsymbol{p}$ together with additional relaxations of the equations in $C_{k}$. The special form for $\boldsymbol{p}^{\lambda}$ we choose is

$$
\boldsymbol{p}^{\lambda}=\sum_{i=1}^{M_{k}} \boldsymbol{a}_{k, i}^{\lambda} \boldsymbol{q}_{i}
$$

with $\boldsymbol{q}: \Omega \times\left\{1, \ldots, M_{k}\right\} \rightarrow \mathbb{R}^{n}$ such that $|\boldsymbol{q}|_{2} \leq 1$ and the vectors $\boldsymbol{a}_{k}^{\lambda} \in \mathbb{R}^{M_{k}}$ which define the Euclidean representation of $d_{k}$; see (5.1). This is only a subset of possible $\boldsymbol{p} \in C_{k}$ in Proposition 5.3. The constraint on $\boldsymbol{p}$ in (5.7) is satisfied, since by the Cauchy-Schwarz inequality and the definition of the representation,

$$
\begin{aligned}
\left|\boldsymbol{p}^{\lambda}-\boldsymbol{p}^{\mu}\right|_{2} & =\left|\sum_{i=1}^{M_{k}}\left(\boldsymbol{a}_{k, i}^{\lambda}-\boldsymbol{a}_{k, i}^{\mu}\right) \boldsymbol{q}_{i}\right|_{2} \\
& \leq \sqrt{\sum_{i=1}^{M_{k}}\left(\boldsymbol{a}_{k, i}^{\lambda}-\boldsymbol{a}_{k, i}^{\mu}\right)^{2}} \cdot \sqrt{\sum_{i=1}^{M_{k}}\left|\boldsymbol{q}_{i}\right|_{2}^{2}} \\
& =\left|A_{k} \boldsymbol{e}^{\lambda}-A_{k} \boldsymbol{e}^{\mu}\right|_{2}|\boldsymbol{q}|_{2} \leq d_{k}(\lambda, \mu) .
\end{aligned}
$$


Plugging (9.28) into (5.7), we obtain the desired result

$$
\begin{aligned}
J_{k}\left(\boldsymbol{v}_{k}\right) & \geq \sup _{|\boldsymbol{q}|_{2} \leq 1}\left\{\sum_{\lambda \in \Lambda_{k}} \int_{\Omega}\left(\sum_{i=1}^{M_{k}} \boldsymbol{a}_{k, i}^{\lambda} \boldsymbol{q}_{i}\right) \cdot \nabla v_{k}^{\lambda} \mathrm{d} x\right\} \\
& =\sup _{|\boldsymbol{q}|_{2} \leq 1}\left\{\int_{\Omega} \sum_{i=1}^{M_{k}} \boldsymbol{q}_{i} \cdot \nabla\left(\sum_{\lambda \in \Lambda_{k}} \boldsymbol{a}_{k, i}^{\lambda} v_{k}^{\lambda}\right) \mathrm{d} x\right\} \\
& =\sup _{|\boldsymbol{q}|_{2} \leq 1}\left\{\int_{\Omega} \sum_{i=1}^{M_{k}} \boldsymbol{q}_{i} \cdot \nabla\left(A_{k} \boldsymbol{v}_{k}\right)_{i} \mathrm{~d} x\right\} \\
& =\mathrm{TV}_{\mathrm{v}}\left(A_{k} \boldsymbol{v}_{k}\right) .
\end{aligned}
$$

The inequality in the first step is a consequence of choosing the special form of $\boldsymbol{p}$ 's, thus reducing the set over which the supremum is taken.

\subsection{Proof of Proposition 6.1.}

Proof. Both $J$ and $R_{\text {data }}$ are support functionals of convex sets in the Hilbert space $\mathcal{L}:=$ $\mathcal{L}^{2}\left(\Omega, \mathbb{R}^{N_{1}+\cdots+N_{d}}\right)$ : (6.3) shows that the regularizer $J$ is the support functional of $K(\mathcal{C})$, while we can see from definition (4.10) that the data term $R_{\text {data }}$ is the support functional of $\mathcal{Q}$. It follows that both $J$ and $R_{\text {data }}$ are lower semicontinuous and convex on $\mathcal{L}$. The set $\mathcal{D}$ is closed; thus its indicator function $\delta_{\mathcal{D}}$ is also convex and closed, and, furthermore, $\delta_{\mathcal{D}}$ is coercive since $\mathcal{D}$ is bounded. From the above, it follows that the functional

$$
\boldsymbol{v} \mapsto J(\boldsymbol{v})+R_{\text {data }}(\boldsymbol{v})+\delta_{\mathcal{D}}(\boldsymbol{v})
$$

is closed and coercive. Since being closed is equivalent to being lower semicontinuous in the Hilbert space topology of $\mathcal{L}$, these properties imply the existence of a minimizer in $\mathcal{L}$ (see Theorems 3.2.5 and 3.3.3 in [3]), which must necessarily lie in $\mathcal{D}$. Since neither functional is strictly convex, the solution is in general not unique.

Acknowledgment. We thank Antonin Chambolle (École Polytechnique) for helpful discussions concerning the proof of Proposition 4.4.

\section{REFERENCES}

[1] G. Alberti, G. Bouchitté, And G. Dal Maso, The calibration method for the Mumford-Shah functional, C. R. Acad. Sci. Paris Sér. I Math., 329 (1999), pp. 249-254.

[2] G. Alberti, G. Bouchitté, and G. Dal Maso, The calibration method for the Mumford-Shah functional and free-discontinuity problems, Calc. Var. Partial Differential Equations, 16 (2003), pp. 299333 .

[3] H. Атtouch, G. Buttazzo, and G. Michaille, Variational Analysis in Sobolev and BV Spaces: Applications to PDEs and Optimization, MOS-SIAM Ser. Optim. 6, SIAM, Philadelphia, 2006.

[4] Y. Boykov And V. Kolmogorov, Computing geodesics and minimal surfaces via graph cuts, in Proceedings of the IEEE International Conference on Computer Vision (ICCV), 2003, pp. 26-33.

[5] Y. Boykov And V. Kolmogorov, An experimental comparison of min-cut/max-flow algorithms for energy minimization in vision, IEEE Trans. Pattern Anal. Mach. Intell., 26 (2004), pp. 1124-1137.

[6] Y. Boykov, O. VeKsler, And R. ZABiH, Fast approximate energy minimization via graph cuts, IEEE Trans. Pattern Anal. Mach. Intell., 23 (2001), pp. 1222-1239. 
[7] T. Brox AND D. Cremers, On local region models and a statistical interpretation of the piecewise smooth Mumford-Shah functional, Int. J. Comput. Vision, 84 (2009), pp. 184-193.

[8] A. Chambolle, Finite-differences discretizations of the Mumford-Shah functional, M2AN Math. Model. Numer. Anal., 33 (1999), pp. 261-288.

[9] A. Chambolle, D. Cremers, and T. Pock, A Convex Approach for Computing Minimal Partitions, Technical Report TR-2008-05, Department of Computer Science, University of Bonn, Bonn, Germany, 2008.

[10] A. Chambolle And T. Pock, A first-order primal-dual algorithm for convex problems with applications to imaging, J. Math. Imaging Vision, 40 (2011), pp. 120-145.

[11] T. F. Chan, S. Esedō̄Lu, And M. Nikolova, Algorithms for finding global minimizers of image segmentation and denoising models, SIAM J. Appl. Math., 66 (2006), pp. 1632-1648.

[12] D. Cremers And K. Kolev, Multiview stereo and silhouette consistency via convex functionals over convex domains, IEEE Trans. Pattern Anal. Mach. Intell., 33 (2010), pp. 1161-1174.

[13] A. Delong, A. Osokin, H. Isack, and Y. Boykov, Fast approximate energy minimization with label costs, Int. J. Comput. Vision, 96 (2012), pp. 1-27.

[14] B. Glocker, N. Paragios, N. Komodakis, G. Tziritas, and N. Navab, Optical flow estimation with uncertainties through dynamic MRFs, in Proceedings of the IEEE International Conference on Computer Vision and Pattern Recognition, 2008.

[15] B. Goldluecke And D. Cremers, Convex relaxation for multilabel problems with product label spaces, in Proceedings of the 11th European Conference on Computer Vision (ECCV), Springer-Verlag, Berlin, Heidelberg, 2010, pp. 225-238.

[16] D. Greig, B. Porteous, And A. Seheult, Exact maximum a posteriori estimation for binary images, J. R. Statist. Soc. B, 51 (1989), pp. 271-279.

[17] H. IsHIKAwA, Exact optimization for Markov random fields with convex priors, IEEE Trans. Pattern Anal. Mach. Intell., 25 (2003), pp. 1333-1336.

[18] M. Klodt, T. Schoenemann, K. Kolev, M. Schikora, and D. Cremers, An experimental comparison of discrete and continuous shape optimization methods, in Proceedings of the 10th European Conference on Computer Vision (ECCV), Springer-Verlag, Berlin, Heidelberg, 2008, pp. 332-345.

[19] V. Kolmogorov, Convergent tree-reweighted message passing for energy minimization, IEEE Trans. Pattern Anal. Mach. Intell., 28 (2006), pp. 1568-1583.

[20] V. Kolmogorov and C. Rother, Minimizing nonsubmodular functions with graph cuts-A review, IEEE Trans. Pattern Anal. Mach. Intell., 29 (2007), pp. 1274-1279.

[21] V. Kolmogorov And R. ZABiH, What energy functions can be minimized via graph cuts, IEEE Trans. Pattern Anal. Mach. Intell., 26 (2004), pp. 147-159.

[22] J. Lellmann, F. BeCKer, AND C. SchnörR, Convex optimization for multi-class image labeling with a novel family of total variation based regularizers, in Proceedings of the IEEE International Conference on Computer Vision (ICCV), 2009.

[23] C. Michelot, A finite algorithm for finding the projection of a point onto the canonical simplex of $\mathbb{R}^{n}$, J. Optim. Theory Appl., 50 (1986), pp. 195-200.

[24] T. Pock And A. Chambolle, Diagonal preconditioning for first order primal-dual algorithms in convex optimization, in Proceedings of the 2011 IEEE International Conference on Computer Vision, 2011.

[25] T. Pock, A. Chambolle, H. Bischof, and D. Cremers, A convex relaxation approach for computing minimal partitions, in Proceedings of the IEEE Conference on Computer Vision and Pattern Recognition (CVPR), 2009, pp. 810-817.

[26] T. Pock, D. Cremers, H. Bischof, and A. Chambolle, An algorithm for minimizing the piecewise smooth Mumford-Shah functional, in Proceedings of the 12th IEEE International Conference on Computer Vision, 2009.

[27] T. Pock, D. Cremers, H. Bischof, and A. Chambolle, Global solutions of variational models with convex regularization, SIAM J. Imaging Sci., 3 (2010), pp. 1122-1145.

[28] T. Pock, T. Schoenemann, G. Graber, H. Bischof, and D. Cremers, A convex formulation of continuous multi-label problems, in Proceedings of the 10th European Conference on Computer Vision (ECCV), Springer-Verlag, Berlin, Heidelberg, 2008, pp. 792-805.

[29] S. Ramalingam, P. Kohli, K. Alahari, And P. Torr, Exact inference in multi-label CRFs with higher order cliques, in Proceedings of the IEEE Conference on Computer Vision and Pattern Recognition, 2008. 
[30] R. T. Rockafellar, Convex Analysis, Princeton University Press, Princeton, NJ, 1996.

[31] D. Schlesinger And B. Flach, Transforming an Arbitrary Min-Sum Problem into a Binary One, Technical report, Dresden University of Technology, Dresden, Germany, 2006.

[32] A. Shekhovtsov, J. Garcia-Arteaga, and T. Werner, A discrete search method for multi-modal non-rigid image registration, in Proceedings of the 2008 IEEE CVPR Workshop on Non-Rigid Shape Analysis and Deformable Image Alignment, 2008.

[33] A. Shekhovtsov, I. Kovtun, and V. Hlavac, Efficient MRF deformation model for non-rigid image matching, Computer Vis. Image Underst., 112 (2008), pp. 91-99.

[34] G. Strang, Maximal flow through a domain, Math. Programming, 26 (1983), pp. 123-143.

[35] E. Strekalovskiy and D. Cremers, Total variation for cyclic structures: Convex relaxation and efficient minimization, in Proceedings of the 2011 IEEE International Conference on Computer Vision and Pattern Recognition, 2011.

[36] E. Strekalovskiy, B. Goldluecke, and D. Cremers, Tight convex relaxations for vector-valued labeling problems, in Proceedings of the 2011 IEEE International Conference on Computer Vision, IEEE Computer Society, Washington, DC, 2011, pp. 2328-2335.

[37] R. Szeliski, Bayesian modeling of uncertainty in low-level vision, Int. J. Comput. Vision, 5 (1990), pp. 271-301.

[38] R. Szeliski, R. Zabih, D. Scharstein, O. Veksler, V. Kolmogorov, A. Agarwala, M. Tappen, AND C. Rother, A comparative study of energy minimization methods for Markov random fields, in Proceedings of the 9th European Conference on Computer Vision (ECCV), Springer-Verlag, Berlin, Heidelberg, 2006, pp. 16-29.

[39] M. TAPpen AND W. Freeman, Comparison of graph cuts with belief propagation for stereo, using identical $M R F$ parameters, in Proceedings of the Ninth IEEE International Conference on Computer Vision, 2003.

[40] M. Wainwright, T. JaAkkola, And A. Willsky, Map estimation via agreement on trees: Messagepassing and linear programming, IEEE Trans. Inform. Theory, 51 (2005), pp. 3697-3717.

[41] C. Zach, D. Gallup, J. Frahm, and M. Niethammer, Fast global labeling for real-time stereo using multiple plane sweeps, in Vision, Modeling and Visualization 2008, Pro Universitate, Berlin, 2008, pp. 243-252.

[42] C. Zach, C. Haene, And M. Pollefeys, What is optimized in tight convex relaxations for multi-label problems?, in Proceedings of the IEEE International Conference on Computer Vision and Pattern Recognition, 2012, pp. 1664-1671.

[43] C. ZACh, T. Pock, And H. Bischof, A duality based approach for realtime TV-L ${ }^{1}$ optical flow, in Proceedings of the 29th DAGM Conference on Pattern Recognition, Springer-Verlag, Berlin, Heidelberg, 2007, pp. 214-223. 